\title{
ON THE LOCAL THEORY OF CONTINUOUS INFINITE PSEUDO GROUPS II*
}

\author{
MASATAKE KURANISHI
}

\section{Chapter III. Differential systems}

In this chapter, we shall formulate, without proof, the theorys of exterior differential systems and of their prolongations using the language in the theory of jets developed by C. Ehresman, because such formulation seems to be most convenient in order to apply the theory to the theory of continuous infinite pseudo-groups, which we shall discuss in the next chapter. Since important theorems in our theory hold only in the real analytic case, we shall exclusively consider the real analytic case. So, we shall omit the adjective "analytic", unless explicitly stated otherwise. As for the fundamental notions in the theory of jets and of differential systems, we refer to [5] and [6], respectively. Detailed proof of the contents of this Chapter will be published in [7].

\section{Differential systems and jets}

Let $M$ be a (real analytic) manifold. Denote by $\Lambda^{h}(M)$ the sheaf of germs of real analytic homogeneous differential forms of degree $h$ on $M . \quad \Lambda(M)$ $=\sum_{h} A^{h}(M)$ is a sheaf of rings. The exterior derivative $d$ induces a mapping $\Lambda^{h}(M) \rightarrow A^{h+1}(M)$. For a sheaf $\Phi$ on $M$ and for an open set $U$ of $M$ denote by $\Gamma(U, \emptyset)$ the set of cross-sections of $\Phi$ over $U$. By a differential system on $M$ we mean a locally finitely generated subsheaf $\Sigma$ of homogeneous ideals in $\Lambda(M)$ such that $d(\Sigma) \subseteq \Sigma$. By locally finitely generated, we mean the following: For any point $w$ in $M$ we can choose an open neighborhood $V$ of $w$ and $f_{1}, \ldots$, $f_{a} \in \Gamma(V, \Sigma)$ such that each $\Sigma_{y}(y \in V)$ is generated as an ideal in $(\Lambda(M))_{y}$ by the germs of $f_{1}, \ldots, f_{a}$ at $w$. Let $B$ be a homogeneous subsheaf of $A(M)$. Then the subsheaf $\Psi$ of ideals generated by $B$ and $d B$ is closed under $d$. Therefore, when $\Psi$ is locally finitely generated, it is a differential system on $M$. If this is so, $\Psi$ is called the differential system generated by $B$. A (not necessarily closed) submanifold $N$ of $M$ is called an integral of a differential

Received January 23, 1961.

* Continuation of my paper with the same title, this J. vol. 15, 1959, pp. 225-260. 
system $\Sigma$, when, for any open set $U$ and for any $\varphi \in I(U, \Sigma)$, the restriction of $\varphi$ to $U \cap N$ is zero, provided $U \cap N$ is not empty. A point $w$ of $M$ is called an integral point of $\Sigma$, when $\varphi(w)=0$ for and $\varphi \in \Sigma_{w}^{[0]}$.

Definition III. 1. A triple $\left(M, M^{\prime}, \pi\right)$ of manifolds $M, M^{\prime}$, and a mapping $\pi$ of $M$ onto $M^{\prime}$ is called a fibered manifold when the rank of the differential $d \pi$ is equal to the dimension of $M^{\prime}$ at each point in $M$.

By a local cross-section $f$ of $\left(M, M^{\prime}, \pi\right)$ we mean a mapping $f$ of an open set $U$ of $M^{\prime}$ into $M$ such that $\pi^{\circ} f$ is the identity mapping of $U$. By a differential system with independent variables, we mean a pair $\left(\Sigma,\left(M, M^{\prime}, \pi\right)\right)$ of a differential system $\Sigma$ on $M$ and a fibered manifold $\left(M, M^{\prime}, \pi\right)$.

Definition III.2. By an integral $f$ of $\left(\Sigma,\left(M, M^{\prime}, \pi\right)\right)$, we mean a local cross-section $f$ of $\left(M, M^{\prime}, \pi\right)$ which is an integral of $\Sigma$ (when $f$ is considered as a submanifold of $M$ ).

We can introduce the notion of differential systems with independent variables which are in involution at integral points (ct. [6]).

Let $\left(M, M^{\prime}, \pi\right)$ be a fibered manifold and $w$ be a point of $M$. If $\left(x_{1}^{\prime}, \ldots\right.$, $\left.x_{n}^{\prime}\right)$ is a coordinate system in $M^{\prime}$ defined on a neighborhood of $\pi(w)$, there is a coordinate system $\left(x_{1}, \ldots, x_{n}, y_{1}, \ldots, y_{m}\right)$ in $M$ defined on a neighborhood of $w$ such that $x_{i}=x_{i}^{\prime} \circ \pi$. Such a coordinate system $(x, y)$ is called a coordinate system in the fibered manifold. In such case, we usually write $x_{i}$ instead of $x_{i}^{\prime}$. Let us assume that $x_{i}(w)=y_{\lambda}(w)=0$, and that $\left\{\left|x_{i}\right|<\varepsilon,\left|y_{\lambda}\right|<\varepsilon\right\}$ is the domain of the coordinate system. Let $S$ be a system of characters (Def. I.2).

Definition III.3. A pair of germs of analytic mappings $\mathbf{F}, \mathbf{F}^{\prime}$ of $\mathscr{A}(S)$ into $\mathscr{H}_{n}^{m}$, of $\mathscr{H}_{n}^{m}$ into $\mathscr{A}(S)$, respectively, is called a parametrization mapping of integrals of $\left(\Sigma,\left(M, M^{\prime}, \pi\right)\right)$ at $w$ in $M$ with respect to the coordinate system $\left(x_{1}, \ldots, x_{n}, y_{1}, \ldots, y_{m}\right)$ in $\left(M, M^{\prime}, \pi\right)$ when they satisfy the following conditions :

(1) $\mathbf{F}^{\prime} \circ \mathbf{F}$ is the identity mapping of $\mathscr{A}(S)$.

(2) If $\mathbf{F}$ is defined at $\xi \in \mathscr{A}(S)$, and if $\left|\left[\mathbf{F}_{\lambda}(\xi)\right](0)\right|<\varepsilon$, then the crosssection on a neighborhood of $\pi(w)$ defined by $y_{\lambda}=\left[\mathrm{F}_{\Lambda}(\xi)\right](x)$ is an integral of $\left(\Sigma,\left(M, M^{\prime}, \pi\right)\right)$.

(3) If $\mathbf{F}^{\prime}$ is defined at $\eta \in \mathscr{\&}_{n}^{m}(u, \varepsilon), \mathbf{F}$ is defined at $\mathbf{F}^{\prime}(\eta)$, and if $y_{\lambda}=\eta_{\lambda}(x)$ is an integral of $\left(\Sigma,\left(M, M^{\prime}, \pi\right)\right)$ on a neighborhood of $\pi(w)$, then $\eta=\mathbf{F}\left(\mathbf{F}^{\prime}(\eta)\right)$. 
In this case, $y_{\lambda}=0$ is an integral of $\left(\Sigma,\left(M, M^{\prime}, \pi\right)\right)$, which is called the center of the parameterization mapping. Let $f$ be an integral of $\left(\Sigma,\left(M, M^{\prime}\right.\right.$, $\pi))$. If a parameterization mapping at $w$ with center $f$ exists with respect to a coordinate $\operatorname{system}(x, y)$, then a parameterization mapping at $w$ with center $f$ exists with respect to any coordinate system $\left(x^{\prime}, y^{\prime}\right)$ such that $f$ is the crosssection $y_{\lambda}^{\prime}=0$. When this is so, the multiplicity and the degree of $\mathscr{H}(S)$ is uniquely determined by $f$ and $\Sigma$.

Theorem III. 1. Let $\left(\Sigma,\left(M, M^{\prime}, \pi\right)\right)$ be a differential system with independent variables. Assume that $\left(\Sigma,\left(M, M^{\prime}, \pi\right)\right)$ is in involution at an integral point $w$. Then there is an integral of $\left(\Sigma,\left(M, M^{\prime}, \pi\right)\right)$ defined on a neighborhood of $w$. For any integral $f$ defined on a suitable open neighborhood of $w^{\prime}$, there is a parametrization mapping at $w$ of integrals of $\left(\Sigma,\left(M, M^{\prime}, \pi\right)\right)$ with center $f$.

Let $\left(M, M^{\prime}, \pi\right)$ be a fibered manifold. Denote by $J^{l}\left(M, M^{\prime}, \pi\right)$ the set of all $l$-jets which can be represented by local cross-sections of $\left(M, M^{\prime}, \pi\right)$. As usual, denote by $j_{x}^{l}(f)$ the $l$-jet at $x$ represented by $f$. We set $\alpha\left(j_{x}^{\prime}(f)\right)=x$, $\beta\left(j_{x}^{l}(f)\right)=f(x) . \quad \alpha \times \beta$ maps $J^{l}\left(M, M^{\prime}, \pi\right)$ to $M^{\prime} \times M$. Clearly $\alpha=\pi \circ \beta$. Let a coordinate system $(x, y)$ be defined on an open set $V$ of $M$. Then $J^{l}\left(M, M^{\prime}\right.$, $\pi)$ has a coordinate system $\left(x_{1}, \ldots, x_{n}, y_{1}, \ldots, y_{m}, \ldots, y_{\Lambda}^{i_{1} . . i_{2}}, \ldots\right)(\lambda=1$, $\ldots, m ; 1 \leq i_{1}, \ldots, i_{\nu} \leq n ; 1 \leq \nu \leq l ; y_{\lambda}^{i_{1} \ldots i_{\nu}}$ symmetric in $i_{1}, \ldots, i_{\nu}$ ) defined on $\beta^{-1}(V)$. Namely, if $X \in \beta^{-1}(V)$ is represented by $f$ at $x$ then

$$
\begin{aligned}
& x_{i}(X)=x_{i}, y_{\lambda}(X)=y_{\lambda}(f(x))=f_{\lambda}(x), \\
& y_{\lambda}^{i_{1} \cdots i_{\nu}}(X)=\frac{\partial^{\nu} f_{\lambda}}{\partial x_{i_{1}} \cdots \partial x_{i \nu}}
\end{aligned}
$$

Thus $J^{l}\left(M, M^{\prime}, \pi\right)$ is a manifold, and $\left(J^{l}, M, \beta\right),\left(J^{l}, M^{\prime}, \alpha\right)$ are fibered manifolds.

Denote by $\rho_{l^{\prime}}^{l}\left(l^{\prime} \leq l\right)$ the canonical projection of $J^{l}\left(M, M^{\prime}, \pi\right)$ onto $J^{l^{\prime}}\left(M, M^{\prime}\right.$, $\pi$ ). In order to avoid inessential complications of notations, let us omit symbols like $\rho \rho^{\prime},\left(\rho_{l^{\prime}}^{l}\right)^{*}$, as far as confusion may not occur. Thus if $g$ is a function on $J^{\prime \prime}, g$ automatically denotes also the function $g^{\circ} \rho^{\prime}$, on $J^{l}$ for any $l \geq l^{\prime}$.

For a local cross-section $f$ of $\left(M, M^{\prime}, \pi\right)$ defined on an open set $U$ of $M$, denote by $j^{\prime}(f)$ the local cross-section of $\left(J^{l}, M^{\prime}, \alpha\right)$ defined by the mapping $U \ni x \rightarrow j_{x}^{l}(f) \in J^{l}$. For an open set $V$ of $J^{l}$, denote by $\Pi^{[1]}(l, V)$ the space of all Pfaffian forms (i) on $V$ such that $j^{l}(f)^{*} \omega=0$ for any local cross-section $f$ 
such that $j^{l}(f) \subseteq V$. If $V^{\prime} \subseteq V$, the restriction mapping sends $\Pi^{[1]}(l, V)$ into $\Pi^{[1]}\left(l, V^{\prime}\right)$. Denote by $I^{[1]}\left(l ;\left(M, M^{\prime}, \pi\right)\right)$, or by $\Pi^{[1]}(l)$ when there is no possibility of confusion, the sheaf of submodules in $A^{1}\left(J^{l}\right)$ defined by the system $\left\{\Pi^{[1]}(l, V)\right\}$.

Proposition III. 1. If $(x, y)$ is a coordinate system in $\left(M, M^{\prime}, \pi\right)$ defined on $U$, and if $V \subseteq \beta^{-1}(U)$, then $\Gamma\left(V, \Pi^{[1]}(l)\right)$ is generated over $\Gamma\left(V, \Lambda^{\circ}\left(J^{l}\right)\right)$ by

$$
\begin{aligned}
\omega_{\lambda} & =d y_{\lambda}-y_{\lambda}^{i} d x_{i} \\
\omega_{\lambda}^{i_{1} \cdots i_{\nu}} & =d y_{\lambda}^{i_{1} \cdots i_{\chi}}-y_{\lambda}^{i_{1} \ldots i_{\searrow} i} d x_{i}
\end{aligned}
$$

$\left(\lambda=1, \ldots, m ; 1 \leq i_{1}, \ldots, i_{\nu}, i \leq n ; 1 \leq \nu \leq l-1\right)$.

Denote by $I\left(l ;\left(M, M^{\prime}, \pi\right)\right)$ the differential system on $J^{l}\left(M, M^{\prime}, \pi\right)$ generated by $I^{[1]}\left(l ;\left(M, M^{\prime}, \pi\right)\right)$.

Proposition III.2. Let $F$ be a local cross-section of $\left(J^{\prime}\left(M, M^{\prime}, \pi\right), M^{\prime}, \alpha\right)$. Then there is a local cross-section $f$ of $\left(M, M^{\prime}, \pi\right)$ such that $F=j^{\prime}(f)$ locally if and only if $F$ is an integral of $I\left(l ;\left(M, M^{\prime}, \pi\right)\right)$.

\section{Partial differential equations}

Let $\left(M, M^{\prime}, \pi\right)$ be a fibered manifold. Set $J^{l}=J^{l}\left(M, M^{\prime}, \pi\right)$.

Definition III.4. By a partial differential equation of order $k$ on $\left(M, M^{\prime}\right.$, $\pi$ ) we mean an open set $U$ of $J^{i}$ and a locally finitely generated subsheaf of ideals $\Phi$ in $A^{\circ}(U)$.

Let $X$ be a point of $U$. We say that $X$ is an integral jet of the partial differential equation $\emptyset$ when $g(X)=0$ for any $g$ in $\Gamma\left(U^{\prime}, \Phi\right)$ and for any open neighborhood $U^{\prime}$ of $X$ in $U$. Denote by $\mathscr{F}^{\circ} \mathscr{\emptyset}$ the set of integral jets of $\emptyset$. Clearly $\mathscr{I}^{\circ} \mathscr{D}$ is a (real) subvariety of $U$. A local crosssection $f$ of $\left(M, M^{\prime}, \pi\right)$ is called an integral of $\Phi$ when $j^{l}(f) \subseteq \mathscr{I}^{\circ} \mathscr{D}$.

The differential system on $U$ generated by $\Phi$ and $\Pi\left(k ;\left(M, M^{\prime}, \pi\right)\right) \mid U$ is called the differential system associated with the partial differential equation $\emptyset$, and will be denoted by $\Sigma(\Phi)$. An integral jet of $\Phi$ is called ordinary when it is an ordinary integral point of $\Sigma(\Phi)$. $\Phi$ is said to be in involution at an integral jet $X$ of $\Phi$ when $\left(\Sigma(\Phi),\left(U, M^{\prime}, \alpha\right)\right)$ is in involution at $X$.

PROposition III.3. Let $f$ be a local cross-section of $\left(M, M^{\prime}, \pi\right)$ such that $j^{l}(f) \subseteq U$. Then $f$ is an integral of $\Phi$ if and only if $j^{l}(f)$ is an integral of 
$\left(\Sigma(\emptyset),\left(U, M^{\prime}, \alpha\right)\right)$. Moreover, for any integral $F$ of $\left(\Sigma(\Phi),\left(U, M^{\prime}, \alpha\right)\right)$, there is a unique integral $f$ of $D$ sush that $F=j^{k}(f)$ locally.

Thus the problem of finding integrals of partial differential equations is equivalent to the problem of finding integrals of the associated differential systems with independent variables. When $\Phi$ is in involution at an integral jet $X$, Theorem 1 shows that there is an integral $f$ of $\emptyset$ such that $X=j_{\alpha(X)}^{k}(f)$ and we can parametrize integrals of $\mathscr{D}$ which are sufficiently near $f$.

Let $\left(x_{1}, \ldots, x_{n}, y_{1}, \ldots, y_{m}\right)=(x, y)$ be a coordinate system in $\left(M, M^{\prime}\right.$, $\pi)$. $J^{l}$ has the coordinate system $\left(x, y, \ldots, y_{\lambda}^{i_{1} \cdots i_{\nu}}, \ldots\right)(\nu \leq l)$ associated with $(x, y)$. Let $g$ be a function defined on an open set $U$ in the domain of the above coordinate system in $J^{l}$. Then $g \circ \rho_{l}^{l+1}$ is a function on $\left(\rho_{l}^{l+1}\right)^{-1}(U)=U^{\prime}$ in $J^{l+1}$. By Proposition 1 , we find easily that $d\left(g \circ \rho_{l}^{l+1}\right) \equiv g^{i} d x_{j}(\bmod . \Pi(l+1$; $\left.\left(M, M^{\prime}, \pi\right)\right)$ ), where $g^{j}$ is a function on $U^{\prime}$. We set $D^{j}(g)=g^{j} . \quad D^{j}$ is linear over the field $\mathbf{R}$ of real numbers, and $D^{j}(g h)=\left(D^{j}(g)\right) \cdot h+g\left(D^{j}(h)\right)$. When $(x, y)$ is changed to $\left(x^{\prime}, y^{\prime}\right)$, we have $d x_{j}=a_{j}^{k} d x_{k}^{\prime}$. Hence

$$
D^{\prime j}(g)=a_{k}^{j} D^{k}(g) \text {. }
$$

Let $F$ be an ideal of $l\left(U, A^{\circ}\left(J^{l}\right)\right)$. Denote by $P(F)$ the ideal in $\left(U^{\prime}, A^{\circ}\left(J^{l+1}\right)\right)$ generated by $F \circ \rho_{l}^{l+1}$ and $D^{j}(g)$, where $j=1, \ldots, n$ and $g$ runs through $F$. The above rule for change of coordinates shows that $P(F)$ is independent of the choice of coordinate system employed for the construction. Let $U$ be an open set of $J^{k}$ and $D$ be a subsheaf of ideals in $A^{\circ}(U)$. For each open set $V \subseteq\left(\rho_{k}^{k+1}\right)^{-1}(U)$, denote by $\Psi(V)$ the ideal in $I^{\prime}\left(V, A^{\circ}\left(J^{k+1}\right)\right)$ generated by the restriction of $P\left(\Gamma\left(\rho_{k}^{k+1}(V), \Phi\right)\right)$. If $V^{\prime} \subseteq V$, the restriction mapping sends $\Psi(V)$ into $\Psi\left(V^{\prime}\right)$. Hence the system $\{\Psi(V)\}$ defines a subsheaf of ideals in $A^{\circ}\left(\left(\rho_{k}^{k+1}\right)^{-1}(U)\right)$. This subsheaf will be denoted by $P(\Phi)$. When $\Phi$ is locally finitely generated, so is $P(\emptyset)$.

Defintion III.j. By the l-th standard prolongation of a partial differential equation $\Phi$ of order $k$, we mean the partial differential equation $P(\cdots(P(\mathscr{D}))$ $\cdots)=P^{l}(\emptyset)$ of order $k+l$, where we operate $P$ l-times.

We immediately have the following proposition for any fixed $l \geq 0$.

Proposition III. 4. A local cross-section $f$ of $\left(M, M^{\prime}, \pi\right)$ is an integral of $\emptyset$ if and only if $f$ is an integral of $P^{l}(\not)$. 
Let $\Phi, \Psi$ be partial differential equations of order $k$ on $\left(M, M^{\prime}, \pi\right)$ defined on open sets $U, V$ in $J^{k}$, respectively. We say that $\Phi \subseteq \Psi$ on a neighborhood of a point $X$ in $J^{k}$, when $X \in U \cap V$ and when there is a neighborhood $W$ of $X$ in $U \cap V$ such that $\Phi_{Y} \subseteq \Psi_{Y}$ for any $Y \in W$.

Theorem III.2. Let $\left(M, M^{\prime}, \pi\right)$ be a fibered manifold. Assume that a partial differential equation $\Phi^{\prime}$ of order $l$ on $\left(M, M^{\prime}, \pi\right)$ is given for any $l \geq l_{0}$. Let $f^{\circ}$ be a cross-section of $\left(M, M^{\prime}, \pi\right)$ over an open neighborhood of a point $x^{\circ}$ in $M^{\prime}$. Assume the following: for any $l \geq l_{0}$,

(1) the restriction of $f^{\circ}$ on a sufficiently small neighborhood of $x^{\circ}$ is an integral of $\Phi^{l}$,

(2) $\Phi^{l+1} \supseteq P\left(\Phi^{l}\right)$ on a neighborhood of $X^{l}=j_{x^{\prime}}^{l}\left(f^{\circ}\right)$,

(3) $X^{l}$ is an ordinary integral jet of $\Phi^{l}$,

(4) for a suitable open neighborhood $U$ of $X^{l_{0}},\left(\mathscr{I}^{\circ} \Phi^{l_{0}} \cap U, \alpha(U), \alpha\right)$ is a fibered manifold,

(5) $\left(\mathscr{F}^{\circ} \Phi^{l+1} \cap V, \mathscr{F}^{\circ} \mathscr{D}^{l} \cap V^{\prime}, \rho_{l}^{l+1}\right)$ is a fibered manifold for suitable open neighborhood $V, V^{\prime}$ of $X^{l+1}, X^{l}$, respectively. Then there is an integer $l_{1}\left(\geq l_{0}\right)$ satisfying the following: $\Phi^{l+1}$ and $P\left(\Phi^{l}\right)$ are equal on a neighborhood of $X^{l+1}$ and $\Phi^{l}$ is in involution at $X^{l}$ for any $l \geq l_{1}$.

Let $U$ be an open set in $J^{l}\left(M, M^{\prime}, \pi\right)$. Let $A$ be a closed submanifold of $U$. Denote by $\Phi(A)$ the subsheaf of ideals in $A^{\circ}(U)$ consisting of germs of functions which are zero on $A . \quad \Phi(A)$ is called the partial differential equation on $\left(M, M^{\prime}, \pi\right)$ associated with $A$. The subvariety $\mathscr{O}^{\circ} \Phi(A)$ of $\left(\rho_{l}^{l+1}\right)^{-1}(U)$ will be denoted by $P(A)$. The following well-known theorem will be used later.

THEOREM III.3. Let $A$ be a closed submanifold of an open set $U$ in $J^{l}(M$, $\left.M^{\prime}, \pi\right)$. Assume that $\left(\emptyset(A),\left(M, M^{\prime}, \pi\right)\right)$ is in involution at any $X$ in $A$. Then $P(A)$ is a closed submanifold of $\left(\rho_{l}^{l+1}\right)^{-1}(U), \emptyset(P(A))=P(\emptyset(A))$, and $(\emptyset(P(A))$, $\left.\left(M, M^{\prime}, \pi\right)\right)$ is in involution at any $(l+1)$-jet in $P(A)$. Moreover $(P(A), A$, $\left.\rho_{l}^{l+1}\right)$ is a fibered manifold with connected fibers.

\section{Chapter IV. Continuous infinite pseudo-groups of E. Cartan}

In this chapter, relations between the continuous infinite pseudo-groups of E. Cartan and the infinite formal Lie $(F)$-groups will be studied. Unfortunately, the relations seem to be not simple. However, when we limit our attention to 
the transitive case, we have a very simple relation. In $\S 1$, we introduce the notion of continuous pseudo-groups of transformations in manifolds. We adopt here a definition which is analytically topological. In so doing, we avoid the direct use of differential systems in involution in the definition, and, in stead, we construct a differential system in involution such that it characterizes the given continuous pseudo-group of transformations and which we shall use in order to develop the theory. We define next the notion of parameter infinite Lie $(F)$-groups of continuous pseudo-groups of transformations, and parameterizing the transformations we prove their existence. In $\$ 2$, depending on the results in $\S 1$, we derive the structure equations of continuous pseudo-groups of transformations. After we introduce the notion of isomorphic prolongations of continuous pseudo-groups of transformations in $\S 3$, we define in $\S 4$ an equivalence relation on the collection of all continuous pseudo-groups of transformations. Continuous infinite pseudo-groups of $\mathrm{E}$. Cartan are then defined as equivalence classes of the relation. Our fundamental result, which will be proved in $\S 5$, states that the transitive continuous pseudo-groups of transformations are in the same class if and only if their parameter infinite Lie $(F)$-groups are isomorphic.

Our description of the theory of continuous infinite pseudo-groups of E. Cartan here is not intended to give a systematic account of the theory, in stead, it is intended to give the definitions and properties which we need to understand the results and proofs in $\$ 5$. By this reason, several important theorems of the theory which are not necessay for our purpose, are omitted. In this chapter, the adjective "real analytic" will be omited as in the preceeding chapter.

$\S 1$. Continuous pseudo-groups of transformations and their parameter formal Lie $(F)$-groups

Let $M$ be a manifold. By a homeomorphism element of $M$ we mean a homeomorphism $f$ of a non-empty open set $U(f)$ in $M$ onto an open set $V(f)$ in $M . \quad U(f)$ and $V(f)$ are called the domain and the range of $f$, respectively. $f^{-1}$ is again a homeomorphism element. If $g$ is another homemorphism element of $M$ and if $U(g) \cap V(f)$ is not empty, we say that the composition of $f$ and $g$ is defined, and the composition $g \circ f$ is a homeomorphism of $f^{-1}(U(g) \cap V(f)$ ) onto $g(U(g) \cap V(f))$. When $U(f) \cap U$ is not empty where $U$ is an open set 
in $M$, we mean by $f \mid U$ the homeomorphism element obtained from $f$ by restricting the domain to $U \cap U(f)$. If $U(g)$ contains $U(f)$ and if $f=g \mid U(f)$, we say that $g$ is an extension of $f$. Let $\varphi$ be a differential form defined on a connected open set $U$ in $M$. If $V(f) \cap U$ is not empty, denote by $f^{*} \varphi$ the differential form on $f^{-1}(V(f) \cap U)$ induced by $f$ from the restriction of $\varphi$ to $V(f) \cap U$. If $\varphi$ and $f^{*} \varphi$ coincide on $f^{-1}(V(f) \cap U) \cap U$, we shall write for simplicity $\varphi=f^{*} \varphi$. Such $\varphi$ is called an invariant differential form of $f$.

Denote by $G^{l}(M)$ the manifold of all invertible $l$-jets of $M$ into $M$. For $X$ in $G^{l}(M)$ denote by $\alpha(X)$ and $\beta(X)$ the source and the target of $X$, respectively. $\left(G^{l}(M), M, \alpha\right)$ and $\left(G^{l}(M), M, \beta\right)$ are fibered manifolds. For any homeomorphism element $f$ of $M, j^{l}(f)=\left\{j_{x}^{l}(f) ; x \in U(f)\right\}$ is a submanifold of $G^{l}(M)$. Denote by $p^{l}(f)$ the homeomorphism element of $G^{l}(M)$ such that its domain is $\alpha^{-1}(U(f))$ and such that, for any $X$ in the domain,

$$
\left[p^{l}(f)\right](X)=X \circ\left(j_{x}^{l}(f)\right)^{-1},
$$

where $x=\alpha(X)$. Clearly $\beta \circ\left(p^{l}(f)\right)=\beta$.

A collection $B$ of homeomorphism elements of $M$ is called a pseudo-group of transformations in $M$, or a pseudo-group in $M$ for simplicity, if it satisfies the followind conditions: $\left(1^{\circ}\right)$ If $f$ is in $\left(B\right.$ then $f^{-1}$ is in $B,\left(2^{\circ}\right)$ if both $f$ and $g$ are in $B$ and if $f \circ g$ can be defined then $f \circ g$ is in $B$. B is called a transitive pseudo-group if for any two points $x$ and $x^{\prime}$ there is an element $f$ in $\mathbb{S}$ such that $f(x)=x^{\prime}$. For $x$ in $M$, denote by $\mathbb{S}_{x}$ the collection of all $f$ in (B) such that $U(f)$ contains $x$ and such that $f(x)=x$. For any two elements $f$ and $g$ in $\mathfrak{G}_{x}$ the composition of $f$ and $g$ is always defined. Denote by $G_{x}^{l}(M)$ the set of all invertible $l$-jets $X$ such that $\alpha(X)=\beta(X)=x . G_{x}^{l}(M)$ is a Lie group. We generally denote by $l^{l}(x)$ the $l$-jet of the identity mapping at $x$. Thus $I^{\prime}(x)$ is the identity element of $G_{x}^{l}(M)$. Denote by $\mathbb{S}_{x}^{l}$ the set of all $X$ in $G_{x}^{l}(M)$ such that there is an element $f$ in $\&$ such that $X=j_{x}^{l}(f) . \quad \mathbb{S}_{x}^{l}$ is a subgroup of $G_{x}^{l}(M)$. Let $M_{1}$ be a manifold. Denote by $U$ an open set in $M$. By a (analytic) family on $U$ in $(B)$, with the parameter manifold $M_{1}$, we mean that, for any point $y$ in $M_{1}$, an element $f^{y}$ in $\&$ is associated in such a way that $U\left(f^{y}\right) \supseteq U$ and that the function $f^{y}(x)$ is real analytic for $(y, x)$ in $\dot{M}_{1} \times U$.

Definition IV.1. A pseudo-group $\mathfrak{B}$ in a manifold $M$ is said to be continuous if it satisfies the following four conditions. 
$\left(1^{\circ}\right)$ There is an integer $l_{1}$ such that, for $l \geq l_{1}, \mathbb{S}_{x}^{l}$ is closed and connected for any $x$ in $M$ and the dimension of $\mathbb{S}_{x}^{l}$ is independent of $x$.

$\left(2^{\circ}\right)$ There is a fibered manifold $\left(M, M^{\prime}, \pi\right)$ such that it satisfies the following conditions:

$\left(2^{\circ} .1\right)$ for any $f$ in $(\mathbb{S}, \pi \circ f=\pi$; for any $x, y$ in $M$ such that $\pi(x)=\pi(y)$, there is $f$ in $(B)$ such that $f(x)=y$;

$\left(2^{\circ} .2\right)$ for any $y$ in $M$, there is an open neighborhood $U$ of $y$ and there is a family $f^{x, x^{\prime}}$ on $U$ in $\mathbb{B}$, with the parameter manifold $U^{\prime}=\left\{\left(x, x^{\prime}\right) ; \pi(x)\right.$ $\left.=\pi\left(x^{\prime}\right)\right\} \subseteq U \times U$, such that $f^{x, x^{\prime}}(x)=x^{\prime}$.

$\left(3^{\circ}\right)$ A homeomorphism element $f$ in $M$ is in $\mathbb{B}$ if and only if, for any connected component $U$ of $U(f), f \mid U$ is in $\$ s$.

$\left(4^{\circ}\right)$ Let $f$ be a homeomorphism element in $M$ such that $U(f)$ is connected. If there is a point $x$ in $U(f)$ such that $f(x)=x$ and $j_{x}^{l}(f) \in \mathbb{S}_{x}^{l}$ for any $l$, then there is an element $g$ in $\$$ which coincides with $f$ on a neighborhood of $x$.

Remark: When $(\$)$ is transitive, $M^{\prime}$ reduces to a point. $\left(M, M^{\prime}, \pi\right)$ is called the fibered manifold of invariants of $\mathbb{S}$.

Definition IV.2. Denote by $A^{l}(\mathbb{B})$ the set of all $X$ in $G^{l}(M)$ such that $X=j_{x}^{l}(f)$ for an element $f$ in 8 .

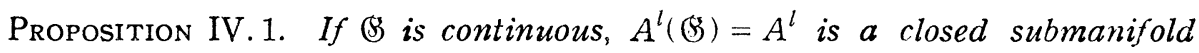
of $G^{l}(M) . \quad \rho_{l}^{l+1}\left(A^{l+1}\right)=A^{l}$. For any $f$ in $\left(S, p^{l}(f)\right.$ preserves $A^{l}$.

Proof. By definitions, it is clear that $p^{l}(f)$ preserves $A^{l}$ for $f$ in $(B$ and that $\rho_{l}^{l+1}\left(A^{l+1}\right)=A^{l}$. To show that $A^{l}$ is a submanifold, we first construct coordinate systems with center $I^{l}\left(x_{0}\right)$. Take small neighborhoods $\mathscr{U}$ of $I^{l}\left(x_{0}\right)$ and $U$ of $x_{0}$ such that, for any $x$ in $U$ and for any $X$ in $G_{x}^{l}(M) \cap \mathscr{U}$, there is a unique one parameter subgroup $X^{t}$ in $G_{x}^{l}(M)$ such that $X^{1}=X$. We can assume that $U$ satisfies the condition $\left(2^{\circ} .2\right)$. Take a submanifold $W$ of $U$ such that $\pi$ induces a bijective mapping of $W$ to an open set in $M^{\prime}$. We can choose $U$ and $W$ such that $\pi(W)=\pi(U)$ and $W \ni x_{0}$. Choose $Y_{1}, \ldots, Y_{d}$ in $\left(\mathbb{S}_{x_{0}}^{l} \cap \mathscr{U}\right.$ such that $\left(t_{1}, \ldots, t_{d}\right) \rightarrow Y_{1}^{t_{1}} \ldots \ldots \circ Y_{d}^{t_{\imath}}$ is a coordinate system with center $I^{l}\left(x_{0}\right)$ in $\mathbb{G}_{z_{0}}^{l}$ for $\left|t_{r}\right|<1$. Take $f_{r}$ in $\mathfrak{B}_{x_{0}}$ such that $Y_{r}=j_{x_{0}}^{l}\left(f^{r_{0}, x_{0}} \circ f_{r}\right)$. Replacing $U$ and $W$ by smaller neighborhoods if necessary, we can assume that $f_{r}$ is defined on $U$ and $f^{x^{\prime}, x}$ is defined on $f_{r}(U)$. For $w$ in $W$, set $Y_{r}(w)=j_{l}^{w}\left(f^{f_{r}}\left(w_{i}, w \circ f_{r}\right)\right.$. 
Clearly $Y_{r}(w) \subseteq \mathbb{S}_{w}^{l}$ and $Y_{r}\left(x_{0}\right)=Y$, If we choose $w$ and $U$ sufficiently small, then $Y_{r}(w) \in \mathbb{S}_{w}^{l} \cap \mathscr{\ell}$ and $Y_{r}(w)^{t} \in \mathbb{S}_{w}^{l}$. Since dimension of $\mathbb{S}_{x}^{l}$ is independent of $x, Y_{1}(w)^{t_{1}} \cdots \cdots Y_{d}(w)^{t_{l}}$ is a coordinate system in $\dot{G}_{w}^{l}$ for $\left|t_{r}\right|<\varepsilon$. Denote by $w(x)$ the point in $W$ such that $\pi(w(x))=\pi(x), w(x)$ is real analytic in $x$. For $\left(x, x^{\prime}\right)$ in $U^{\prime}$ and $\left(t_{1}, \ldots, t_{d}\right)$, set

$$
\begin{aligned}
\lambda\left(x, x^{\prime}, t_{1}, \ldots, t_{d}\right) & \\
& =j_{w(x)}^{l}\left(f^{w(x), x^{\prime}}\right) \circ Y_{1}(w(x))^{t_{1}} \circ \ldots \circ Y_{d}\left(w^{\prime}(x)\right)^{t} \circ j_{w(x)}^{l}\left(f^{w_{(x)}, \mathfrak{r}}\right)^{-1}
\end{aligned}
$$

Clearly, $\lambda$ maps $U^{\prime} \times\{|t|<,\varepsilon\}$ real analytically onto an open set in $A^{\prime}$. For any $X_{0}$ in $A^{l}$, take $f$ in $\left(S\right.$ such that $X_{0}=j_{x_{0}}^{l}(f)^{-1}$ where $x_{0}=\beta\left(X_{0}\right)$. Then $p^{l}(f)$ maps $I^{l}\left(x_{0}\right)$ to $X_{0}$. Thus $p^{l}(f) \circ \lambda$ maps $U^{\prime} \times\left\{\left|t_{r}\right|<\varepsilon\right\}$ real analytically onto an open neighborhood of $X_{0}$ in $A^{l}$, because $p^{l}(f)$ induces a homeomorphism element of $A^{l}$. It is clear that these coordinate systems are related to each other real analytically. Thus $A^{l}$ is a real analytic submanifold of $G^{l}(M)$. It remains to show that $A^{l}$ is closed. Take a sequence $X_{n}$ in $A^{l}$ converging to $X$. Since $\pi\left(\alpha\left(X_{n}\right)\right)=\pi\left(\beta\left(X_{n}\right)\right)$, it follows that $\pi(\alpha(X))=\pi(\beta(X))$. Therefore there is $f$ in $(S)$ such that $f(\alpha(X))=\beta(X)$. Considering $j_{3(X)}^{l}\left(f^{-1}\right) \circ X$ instead of $X$, we can assume without loss of generality that $X$ is in $G_{x}^{l}(M)$. By a similar construction as that of the coordinate mapping $\lambda$, we show easily that there is a homeomorphism of $U^{\prime} \times G_{x}^{l}(M)$ onto $(\alpha \times \beta)^{-1}\left(U^{\prime}\right)\left(\subseteq G^{l}(M)\right)$ which sends $U^{\prime} \times \mathbb{G}_{x}^{l}$ onto $A^{l} \cap(\alpha \times \beta)^{-1}(\bar{U} \times U)$, where $U$ is a sufficiently small neighborhood of $x$ and $U^{\prime}=\left\{\left(y, y^{\prime}\right) ; \pi(y)=\pi\left(y^{\prime}\right)\right\} \subseteq U \times U$. On the other hand $\mathbb{G}_{x}^{l}$ is closed in $G_{x}^{l}(M)$. Therefore $A^{l} \cap(\alpha \times \beta)^{-1}(U \times U)$ is closed in $(\alpha \times \beta)^{-1}(U \times U)$, and so $X$ is in $A^{l}$. Thus $A^{l}$ is closed and this finishes the proof.

Denote by $\rho_{1}$ and $\rho_{2}$ the projections of $M \times M$ to the first and the second factors, respectively. $\left(M \times M, M, \rho_{1}\right)$ is a fibered manifold. For any homeomorphism element $f$ of $M, U(f) \ni x \rightarrow(x, f(x)) \in M \times M$ is a local cross-section $f^{\prime}$ of $\left(M \times M, M, \rho_{1}\right)$. By mapping $j_{x}^{\prime}(f)$ to $j_{x}^{l}\left(f^{\prime}\right)$, we have a canonical injection of $G^{l}(M)$ into $J^{l}\left(M \times M, M, \rho_{1}\right)$ (cf. $\S 1$, Chapt. III). We will identify $G^{l}(M)$

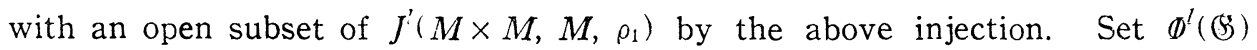
$=\Phi\left(A^{i}((3))\right.$, the partial differential equation on $\left(M \times M, M, \rho_{1}\right)$ associated with the closed submanifold $A^{l}(\mathbb{3})$ of the open set $G^{l}(M)$ in $J^{l}\left(M \times M, M ; \rho_{1}\right)$ (cf. the end of Chapt. III).

Proposition IV.2. $A^{l+1}(B) \subseteq P\left(A^{l}(B)\right)$, or equivalently, 


$$
\mathscr{D}^{l+1}\left(\left(\xi^{i}\right) \supseteq P\left(\phi^{\prime}((\$))\right)\right.
$$

Froof. Take $X_{0}$ in $A^{l}$. Let $u^{\prime}$ be a function defined on an open neighborhood $U$ of $X_{0}$ such that $w=0$ on $A^{l} \cap U$. Take $f$ in $\&$ such that $X_{0}=i_{x_{0}}^{l}(f)$. Then $w\left(j_{x}^{l}(f)\right)=0$. We have $d\left(w \circ \rho_{l}^{l+1}\right) \equiv\left(D^{j}(w)\right) d x_{j}\left(\bmod \Pi\left(l+1 ;\left(M \times M, M, \rho_{1}\right)\right)\right)$. Since $I(l+1)$ and $d\left(w 0^{\circ} l^{i+1}\right)$ vanish on the submanifold $j^{l-1}(f)$, we have $\left[D^{j}(w)\right]\left(j_{x}^{l+1}(f)\right)=0$. Hence $D^{j} w=0$ on $\left(\rho_{l}^{l+1}\right)^{-1}(U) \cap A^{l+1}$. Thus $\left.\mathscr{D}^{l+1}(\mathcal{S})\right)$ $\supseteq P\left(\mathscr{D}^{l}((\xi))\right.$.

Definition IV.3. Let $\mathbb{B}$ be a pseudo-group in $M$. Denote by $\mathbb{S}^{-}$the collection of all honeomorphism element $f$ in $M$ such that for any $x$ in $U(f)$ there is an elenent $g$ in $(B)$ such that $f$ and $g$ coincide on a neighborhood of $x$. It is clear that $\mathrm{B}^{-}$is again a pseudo-group in $M . \mathrm{B}^{-}$is called the completion of (3). When $B=\mathrm{B}^{-}$, we say that $\mathrm{B}$ is complete.

It is easy to verify that $\mathbb{B}^{-}$is continuous if and only if $B$ is continuous.

Theorem IV.1. Let (\$s be a continuous pseudo-group in $M$. Take $x$ in $M$. Then there is an open neighborhood $U$ of $x$ such that for an integer $l_{1}=l_{1}(x)$ the followings are satisfied:

$\left(1^{\circ}\right)$ For any fixed $l \geq l_{1}$, a homeomorphism elemen $f$ of $M$ sush that $U(f) \subseteq U$ is in $\mathbb{B}^{-}$if and only if $j^{\prime}(f)$ is in $A^{l}(\mathbb{B})$,

$\left(2^{\circ}\right) \quad A^{l+1}(\mathbb{B}) \cap \alpha^{-1}(U)=P\left(A^{l}(\$)\right) \cap \alpha^{-1}(U)$ for any $l \geq l_{1}$,

$\left(3^{\circ}\right)\left(\Phi^{\prime}(\$),\left(M \times M, M, \rho_{1}\right)\right)$ is in involution at any $X$ in $A^{\prime}(\$) \cap \alpha^{-1}(U)$ for any $l \geq l_{1}$.

Proof. Denote by $i$ the identity mapping of $M$. Setting $f^{\lrcorner}=$the graph of $i, x^{\circ}=x, \mathscr{D}^{l}=\mathscr{D}^{\prime}(\mathbb{B})$ in Theorem III.2., we find that $\mathscr{D}^{l}(\mathscr{S})$ is in involution at $I^{\prime}\left(x^{\prime}\right)$ and $\mathscr{J}^{l+1}(\xi)=P\left(\Phi^{\prime}((\xi))\right.$ on a neighborhood of $I^{l+1}\left(x^{\prime}\right)$ for $x^{\prime}$ sufficiently near $x$ and for sufficiently large $l$. Take a neighborhood $U$ of $x$ and $l_{1}$ such that the above holds for $x^{\prime} \in U$ and for $l \geqq l_{l}$. Take $X$ in $A^{l}$ such that $\beta(X)$ $=x^{\prime}$ is in $U$. Choose $f$ in $B$ such that $X=j_{r^{\prime}}^{l}(f)^{-1}$. Then $p^{l}(f)$ induces an isomorphism of $\mathscr{D}^{l}\left((B)\right.$ at $X$ to $\mathscr{D}^{l}(\mathbb{B})$ at $I^{l}\left(X^{\prime}\right)$. Therefore $\mathscr{D}^{\prime}(\mathbb{B})$ is in involution at $X$, and $A^{l+1}(\$)$ and $P\left(A^{l}(\$)\right)$ coincide on a neighborhood of $X$. On the other hand, the mapping $Y \rightarrow Y^{-1}$ induces a homeomorphism of $A^{\prime}((\$)$ onto itself. Therefore $\left(2^{\circ}\right)$ and $\left(3^{\circ}\right)$ hold for $U$ and $l$.

By the definition of $A^{l}\left((B)\right.$, it is clear that $j^{l}(f)$ is in $A^{l}(\dot{B})$ for $f$ in (3). Assume conversely that $j^{l}(f)$ is in $A^{\prime}\left((\$)\right.$ for an integer $l \geq l_{1}$ and that $U(f)$ 
is in $U$. Then by $\left(2^{c}\right), j^{\prime \prime}(f)$ is in $A^{l^{\prime}}(\mathbb{B})$ for any $l^{\prime} \geq l$. We will show that there is $g$ in $\&$ such that $f$ and $g$ coincide on a neighborhood of any fixed $x_{3}$ in $U$. Take $h$ in $\&$ such that $h\left(f\left(x_{0}\right)\right)=x_{0}$ (cf. the condition (2.1) in Def. IV.1.). Since $A^{l^{\prime}}(\mathbb{S})$ is preserved under $p^{l^{\prime}}\left(h^{-1}\right), j^{l^{\prime}}(f \circ h)$ is also in $A^{l^{\prime}}(\mathbb{B})$. Since $f \circ h\left(x^{\prime}\right)=x^{\prime}$, where $x^{\prime}=f\left(x_{0}\right)$, and $A^{l^{\prime}}(\&) \cap G_{x^{\prime}}^{l^{\prime}}(M)=\mathbb{S}_{x^{\prime}}^{l^{\prime}}$, the condition $\left(4^{c}\right)$ in Def. IV.1. implies that there is $g^{\prime}$ in $\&$ such that $f \circ h=g^{\prime}$ on a neighborhood of $x^{\prime}$. Thus $g^{\prime} \circ h^{-1}$ in $(S)$ and $f$ coincide on a neighborhood of $x_{0}$. Therefore (10) holds.

Conversely we have the following:

Theorem IV.2. Let $\$$ be a pseudo-group in a manifold $M$. Assume that there is a closed submanifold $A$ of $G^{l}(M)$ for an integer $l$ satisfying the following conditions :

$\left(1^{\circ}\right) \quad A \cap G_{x}^{l}(M)$ is a (non-empty) subgroup of $G_{x}^{l}(M)$ for any $x$ in $M$;

$\left(2^{\circ}\right)\left(\Phi(A),\left(M \times M, M, \rho_{1}\right)\right)$ is in involution at $I^{l}(x)$ for any $x$ in $M$, where $\rho_{1}$ is the projection to the first factor;

$\left(3^{\circ}\right)$ there is a closed submanifold $N$ of $M \times M$ such that $(A, N, \alpha \times \beta)$ is a fibered manifold and each of its fiber is connected;

$\left(4^{\circ}\right)\left(N, M, \rho_{1}\right)$ is a fibered manifold and each of its fiber is connected;

$\left(5^{\circ}\right)$ a homemorphism element $f$ of $M$ is in $B$ if and only if $j^{\prime}(f)$ is in $A$. Then $(B)$ is a continuous pseudo-group in $M$ and $A=A^{l}$ (B).

We first prove the following lemma :

Lemma VI. 1. Let $(B$ be a pseudo-group in a manifold $M$. Assume the following for an integer $l$;

( $\left.1^{\circ}\right) \quad A=A^{l}(B)$ is a closed submanifold of $G^{l}(M)$;

$\left(2^{\circ}\right) \quad A \cap G_{x}^{l}(M)$ is a (non-empty) connected subgroup of $G_{x}^{l}(M)$ for any $x$ in $M$;

$\left(3^{\circ}\right)\left(\emptyset(A),\left(M \times M, M, \rho_{1}\right)\right)$ is in involution at any $X$ in $A$;

(4 $4^{\circ}$ a homeomorphism element $f$ of $M$ is in $\&$ if and only if $j^{\prime}(f)$ is in $A$. Then the same conditions are satisfied for any $l^{\prime} \geq l$. Moreover $A^{l+h}(\mathbb{B})=P^{h}(A)$.

Proof. Clearly it is sufficient to prove the case $l^{\prime}=l+1$. By Theorem III. 3., $P(A)$ is a closed submanifold of $G^{l+1}(M)$. By the same theorem ( $\left.\Phi(P(A)),\left(M \times M, M, \rho_{1}\right)\right)$ is in involution at any $Z$ in $P(A)$. Therefore there is an integral $F$ of $\left(\Phi(P(A)),\left(M \times M, M, \rho_{1}\right)\right)$ such that $Z=j_{\alpha(Z)}^{l+1}(F)$. Hence 
by $\left(4^{\circ}\right)$ and Prop. III. 4 there is $f$ in $(\$)$ such that $Z=j_{\alpha(Z)}^{l+1}(f)$. Since $A^{l+1}(\&)$ $\subseteq P(A)$ by Prop. IV.2, it follows that $P(A)=A^{l+1}(\mathbb{S})$. In particular, $A^{l+1}(\mathbb{B})$ is a closed submanifold of $G^{l+1}(M)$. The last conclusion in Theorem III. 3 implies that $A^{l+1}(\mathbb{B}) \cap G_{x}^{l+1}(M)$ is connected. Since $P(A)=A^{l+1}(\$),\left(\Phi\left(A^{l+1}(\mathbb{B})\right)\right.$, $\left.\left(M \times M, M, \rho_{1}\right)\right)$ is in involution at any $Z$ in $A^{l+1}(\mathbb{B})$ by Theorem III.3. The condition $\left(4^{\circ}\right)$ for $l+1$ is also satisfied by Prop. III. 4.. This finishes the proof of the lemma.

Proof of Theorem 2. Denote by $N^{\prime}$ the set of all $(x, y)$ in $M \times M$ such that $f(x)=y$ for an element $f$ in (B. Set $N_{x}=\{y \in M ; \quad(x, y) \in N\}, N_{x}^{\prime}$ $=\left\{y \in M ; \quad(x, y) \in N^{\prime}\right\} . \quad$ By $\left(5^{\circ}\right) N_{x}^{\prime} \subseteq N$, and by $\left(4^{\circ}\right) N_{x}$ is a connected manifold. $\left(2^{\circ}\right)$ and $\left(5^{\circ}\right)$ imply that there is a neighborhood $U_{x}$ of $x$ in $N_{x}$ such that for any $w$ in $U_{x}$ there is $f_{x}^{w}$ in $\&$ such that $f_{x}^{w}(x)=w$. Take $y$ in $N_{x}^{\prime}$ and $f$ in $\&$ such that $f(x)=y$. Then $f_{y}^{w} \circ f(x)=w$, and so $U_{y} \subseteq N_{x}^{\prime} \subseteq N_{x}$. Since $\operatorname{dim} N_{x}=\operatorname{dim} N_{y}, U_{y}$ is a neighborhood of $y$ in $N_{x}^{\prime}$. Therefore $N_{x}^{\prime}$ is open in $N_{x}$. If $y$ is not in $N_{x}^{\prime}$, the sarne argument shows that $N_{x}^{\prime} \cap U_{y}$ is empty. Hence $N_{x}^{\prime}$ is closed in $N_{x}$. Since $N_{x}$ is connected by $\left(4^{\circ}\right)$ and $N_{x}^{\prime}$ is not empty, we have $N_{x}^{\prime}=N_{x}$. Therefore, $N=N^{\prime}$.

Set $A^{\prime}=A^{l}\left((\Im)\right.$. We will show that $A^{\prime}=A$. By $\left(5^{\circ}\right) A^{\prime} \subseteq A$. Since $I^{\prime}(x)$ $\in A$ by $\left(1^{\circ}\right)$, the identity mapping of $M$ is in $\mathcal{B}$. Hence $I^{l}(x) \in A^{\prime}$. $\quad\left(2^{\circ}\right)$ implies that, for any $X$ in $A$ sufficiently near $I^{\prime}(x)$, there is an integral $F$ of the partial differential eqution $\left(\mathscr{D}(A),\left(M \times M, M, \rho_{1}\right)\right)$ such that $F(\alpha(X))=X$. Therefore, by $\left(5^{\circ}\right)$, there is $f \in \mathbb{S}$ such that $X=j_{\propto(X)}^{l}(f)$. Hence $A=A^{\prime}$ on a neighborhood of $I^{l}(x)$. Since $A \cap G_{x}^{l}(M)$ is a connected subgroup by $\left(1^{\circ}\right)$ and $\left(3^{\circ}\right)$, and coincide with a subgroup $A^{\prime} \cap G_{x}^{l}(M)$ on a neighborhood of $I^{l}(x)$, it follows that $A \cap G_{x}^{l}(M)=A^{\prime} \cap G_{x}^{l}(M)$ for any $x$ in $M$. Now, take any $X$ in $A$. Since $(\alpha(X), \beta(X))=(x, y)$ is in $N=N^{\prime}$, there is $f$ in (f) such that $f(x)=y$. Then $p^{\prime}\left(f^{-1}\right)$ induces a homemorphism of $A^{\prime} \cap G_{y}^{l}(M)$ onto $A^{\prime} \cap G_{x y}^{\prime}(M)$, where $G_{x y}^{l}(M)=(\alpha \times \beta)^{-1}(x, y)$. Since $A^{\prime} \cap G_{y}^{l}(M)=A \cap G_{y}^{l}(M)$ and $A \cap G_{l}^{x y}(M)$ are manifolds of the same dimensions by $\left(3^{\circ}\right)$, it follows that $A^{\prime} \cap G_{x y}^{\prime}(M)$ is an open submanifold of $A \cap G_{x y}^{l}(M)$. Since $p^{l}\left(f^{-1}\right)$ induces a homeomorphism of $G_{y}^{l}(M)$ onto $G_{x y}^{l}(M)$, and $A^{\prime} \cap G_{y}^{l}(M)$ is closed in $G_{y}^{l}(M)$, its image $A^{\prime} \cap G_{x y}^{l}(M)$ is closed in $G_{x y}^{\prime}(M)$ and so is closed in $A \cap G_{x y}^{l}(M)$. Since $A \cap G_{x y}^{l}(M)$ is connected by $\left(3^{\prime}\right)$, it follows that $A \cap G_{x, y}^{l}(M)=A^{\prime} \cap G_{x y}^{l}(M)$. In particular $X$ is in $A^{\prime}$, Therefore $A=A^{l}($ (S) $)$, 
Since $A=A^{l}(\xi)$, for any $X$ in $A$ there is $f$ in $B$ such that $p^{l}\left(f\left(\left(I^{l}(x)\right)\right.\right.$ $X$, where $x=\beta(X)$. Because $p^{l}(f)$ is a homeomorphism element of $A=A^{l}(B)$, $p^{l}(f)$ induces an isomorphism of $\Phi(A)$ at $X$ onto $\Phi(A)$ at $I^{l}(x)$. Therefore, $\left(\mathscr{D}(A),\left(M \times M, M, \rho_{1}\right)\right)$ is in involution at any $X$ in $A$. Thus the condition in Lemma IV. 1 is satisfied for $(B)$. Therefore $(B)_{x}^{\prime \prime}$ is a connected subgroup for any $l^{\prime} \geq l$. Since $A^{l+h^{\prime}}(\mathbb{S})=P^{h}(A)$, the last conclusion of Theorem III. 3 implies that $\operatorname{dim}\left(S_{x}^{\prime \prime}\right.$ is independent of $x$. Thus the condition $\left(1^{\circ}\right)$ in Def. IV. 1 is satisfied for $(\mathfrak{B}$.

In order to show that the condition $\left(2^{\circ}\right)$ in Def. IV. 1 is satisfied for $\mathbb{G}$, we construct a fibered manifold $\left(M, M^{\prime}, \pi\right)$ of invariants of $\$ B$. We say that $x$ is equivalent to $y$ when $(x, y)$ is in $N^{\prime}$. Clearly this is an equivalence relation. Denote by $[x]$ the class of $x$. Let $M^{\prime}$ be the set of classes under this equivalence relation. Since $N=N^{\prime},[x]=[y]$ if and only if $(x, y)$ is in $N$. Hence $N_{x}$ is the set of points $y$ such that $[x]=[y]$. By $\left(4^{\circ}\right), N_{x}$ is a closed submanifold of $M$. Take a (not necessarily closed) submanifold $W$ in $M$ passing through $x$ such that the tangent vector spaces to $W$ and to $N_{x}$ at $x$ intersect only at the origin and such that they generate the tangent vector space to $M$. Take a coordinate system $\left(w_{i}, \ldots, w_{m}\right)$ in $W$ with center $x$. We define a coordinate system in $M^{\prime}$ by $\left[\left(w_{1}, \ldots, w_{m}\right)\right]$. Since $N_{x}$ is connected by $\left(4^{\circ}\right)$, these coordinates systems at $[x]$ are related real analytically when we change the representative $x$ and the coordinate system $(w)$. Then it will be clear that these coordinate systems are related real analytically when we change $[x]$. Thus $M^{\prime}$ is a manifold. Set $\pi(x)=[x]$. Then it is clear that $\left(M, M^{\prime}, \pi\right)$ is a fibered manifold and the condition $\left(2^{\circ} .1\right)$ in Def. IV.1 is satisfied for $\$$. The condition $\left(2^{\circ} .2\right)$ follows from our assumption $\left(2^{\circ}\right)$. The verification of the condition $\left(3^{c}\right)$ in Def. IV, 1 is trivial. Assume now that $f$ satisfies the assumption in $\left(4^{\mathrm{c}}\right)$ in Def. IV.1. Since $\left.A^{l+h}\right)(B)=P^{h}\left(A^{l}(\$)\right)$ for any $h \geq 0$, and $A^{l+h}(\$)$ is a real analytic submanifold, it follows that $j^{l}(f)$ is in $A^{l}$. Therefore $f$ is in (3) by our assumption $\left(5^{\circ}\right)$. Thus we proved that $B$ is continuous.

Definition IV.4. A pair $(\mathfrak{G}, p)$ of a continuous pseudo-group (\$S on a manifold $M$ and of a point $p$ in $M$ is called a local continuous pseudo-group in $M$ at $\mathrm{p}$.

In order to introduce the notion of parameter infinite Lie $(F)$-groups of local continuous pseudo-groups, we make the following preliminaries. Let $G$. 
be a formal Lie $(F)$-group over the field $\mathbf{R}$ of real numbers (cf. Chapt. II). We say that $G$ is an infinite Lie $(F)$-group, when the parameter space of $G$ is $H^{T}(S)$ for a system of characters $S$ and when $G$ is the formalization of germs of analytic mappings of $\mathscr{Z}^{R}(S)+\mathscr{H}^{R}(S)$ into $\mathscr{L}^{R}(S)$ (cf. the end of $\$ 4$ Chapt. I). By an infinite Lie $(F)$-group $G$, we often mean also the germ of analytic mappings of $\mathscr{H}^{R}(S)+\mathscr{H}^{R}(S)$ into $\mathscr{H}^{R}(S)$ determined by the formal mapping $G$, and so often use the bold letter $\mathbf{G}$ instead of $G$. The degree and the multiplicity of $H^{R}(S)$ are called the degree and the multiplicity of $G$, respectively. The germ of analytic mappings of $\mathscr{I}_{n}^{n n}+\mathscr{\varkappa}_{n}^{n_{R}}$ into $\mathscr{\Psi}_{n}^{n R}$ determined by $\mathscr{M}_{u}$ in $(C) \S j$ Chapt. I is an infinite Lie $(F)$-group, which is called the general infinite Lie $(F)$-group in $n$-dimensional space. We denote it by $\operatorname{GIL}(n)$.

Definition IV.5. An infinite Lie $(F)$-group $\mathbf{G}$ is called a parameter infinite Lie $(F)$ group, or a parameter group, in short, of a local continuous pseudogroup $(\mathfrak{G}, \mathfrak{p})$ in $M$ if there is a coordinate neighborhood $U=\left\{\left(x_{1}, \ldots, x_{i 2}\right)\right.$; $\left.\left|x_{i}\right|<\varepsilon\right\}$ with center $\mathfrak{p}$ in $M$ with the following conditions:

$\left(1^{\circ}\right)$ There is a germ of analytic mappings $\mathbf{F}$ of the parameter space $=\mathscr{H}^{R}(S)$ of $\mathbf{G}$ into $\mathbb{H}_{n}^{n R}$ such that $\mathbf{F}$ is a representation of $\mathbf{G}$ into $\operatorname{GIL}(n)$; there is a germ $\mathbf{F}^{\prime}$ of analytic mappings of $\mathscr{Y}_{n^{n}}^{n R}$ into $\mathscr{Y}^{R}(S)$ such that $\mathbf{F}^{\prime} \circ \mathbf{F}$ is the identity mapping.

$\left(2^{\circ}\right)$ There are strictily positive real numbers $u_{1}$ and $a$, and an integer $k$ such that $\mathbf{F}$ is defined on $y^{R}\left(u, u^{k} a\right)$ for any $u \leq u_{1}$ and such that $\mathbf{F}$ satisfies the following conditions: If $\xi$ is in $\mathscr{H}^{I}\left(S ; u, u^{k} a\right)$ for a real number $u \leq u_{1}$, there is a homeomorphism element $f$ in $\&$ such that $U(f)$ contains $\mathfrak{p}$ and such that $f$ coincides with $f^{\mathrm{F} \mid 5 !}$ on a neighborhood of $\mathfrak{p}$, where we identified $U$ uith a domain in $\mathbf{R}^{\prime \prime}$ by the coordinate system $(x)$ and $f^{\mathrm{F}()}$ is the homeomorphism element in $U$ defined in (C) $\$ 5$, Chapt. I.

$\left(3^{\circ}\right)$ There are strictiy positive real numbers $u_{1}^{\prime}$ and $a^{\prime}$, and an integer $k^{\prime}$ with the following conditions: If $\eta$ is in $\mathscr{H}_{n}^{n i}\left(u, u^{k^{\prime}} a^{\prime}\right)$ for a real number $u \leq u_{1}^{\prime}$ and if $f^{\prime \prime}$ coincides with a homeomorphism element $f$ in $(5)$ on a neighborhood of p then $\left(\mathbf{F}\left(\mathbf{F}^{\prime}(\eta)\right)=\eta\right.$.

Definition IV.6. The collection of the coordinate system $(x)$ on $U, \mathbf{F}$, and $\mathbf{F}^{\prime}$ as in Def. IV. 5 will be called a realization of $\mathbf{G}$ as $((\mathcal{B}, \mathfrak{D})$. 
From the definition it follow's immediately the following:

Proposition IV.3. A collection $\left\{U, \mathbf{F}, \mathbf{F}^{\prime}\right\}$ is a realization of $(\mathcal{B}, \mathcal{p})$ if and only if it is a realization of $\left(\mathbb{S}^{-}, \mathfrak{p}\right)$.

THeORem IV.3. Let $(\$, p)$ be a continuous local pseudo-group in $M$. Then there is a parameter infinite Lie $(F)$-group of $(\mathfrak{S}, \mathfrak{p})$.

Proof. Take an integer $l$ so large that the contention in Thereom IV. 1 holds. Take a coordinate system $\left(x_{1}, \ldots, x_{n}\right)$ in $M$ with center $\mathfrak{p}$. Take a coordinate system of the form $x_{1} \circ \rho_{1}, \ldots, x_{n} \circ \rho_{1}, x_{1} \circ \rho_{2}-x_{1} \circ \rho_{1}, \ldots, x_{n} \circ \rho_{2}$ $-x_{n} \circ \rho_{1}, v_{1}, \ldots, v_{a}$ in $J^{l}\left(M \times M, M, \rho_{1}\right)$ with center $I^{l}(\mathfrak{p})$, where $\rho_{1}$ and $\rho_{2}$ are the canonical projections to the first and the second factors of $M \times M$. Remark that we identified $G^{l}(M)$ with an open subset of $J^{l}\left(M \times M, M, \rho_{1}\right)$. We choose the coordinate system such that $j^{l}(i)$ is represented by $x_{j} \circ \beta_{2}-x_{j} \circ \rho_{1}=v_{1}=v_{2}$ $=\cdots=0$, where $i$ is the identity mapping of $M$. Since $\left(\Phi^{l}(\$),(M \times M, M\right.$, $\left.\rho_{1}\right)$ ) is involution at $I^{l}(\mathfrak{p})$ by Theorem IV. 1 , there is a parameterization mapping of integrals of $\left(\Sigma\left(\Phi^{l}(\$)\right), G^{l}(M), \alpha\right)$ at $I^{l}(p)$ with respect to the coordinate system, i.e. there is a system of characters $S$ and a pair of germs of real analytic mappings $\mathbf{F}^{-}, \mathbf{F}^{-1}$ of $\mathscr{H}^{R}(S)$ into $\mathscr{H}_{n}^{m R}$ and of $\mathscr{H}_{n}^{m R}$ into $\mathscr{H}^{R}(S)$ which satisfy the condition in Def. III. 3, where $m+n$ is the dimension of $G^{l}(M)$. $\eta$ in $\mathscr{\&}_{n}^{n R}$ can be considered as a local cross-section $x \rightarrow(x, x+\eta(x))$ of $\left(M \times M, M, \rho_{1}\right)$. Also $\zeta$ in $\mathscr{H}_{n}^{m R}$ can be considered as a local cross-section of $\left(G^{l}(M), M, \alpha\right)$ in terms of the coordinate system $\left(x \circ \rho_{1}, x \circ \rho_{2}-x^{\circ} \rho_{1}, v_{1}, \ldots\right)$. Using this identification, $j^{l}(\eta)$ in $\mathscr{C}_{n}^{m R}$ is defined for $\eta$ in $\mathscr{Z}_{n}^{n R}$, and $\alpha \times \beta(\zeta)$ in $\mathscr{Y}^{n R}{ }_{n}^{n R}$ for $\zeta$ in $\mathscr{H}_{n}^{m R}$. We have $[\alpha \times \beta(\zeta)]_{j}(x)=\zeta_{j}(x)$ for $j=1, \ldots, n$. Set $\mathbf{F}(\xi)=\alpha \times \beta\left(\mathbf{F}^{-}(\xi)\right)$ for $\xi$ in $\mathscr{H}^{R}(S)$, and $\mathbf{F}^{\prime}(\eta)=\mathbf{F}^{-}\left(j^{\prime}(\eta)\right)$ for $\eta$ in $\mathscr{H}^{\prime}{ }_{n}^{n R}$, provided they have meanings. $\quad \mathbf{F}$ and $\mathbf{F}^{\prime}$ are germs of analytic mappings of $\mathscr{X}^{R}(S)$ into $\mathscr{Y}_{n}^{n R}$ and of $\mathscr{H}_{n}^{n R}$ into $\mathscr{S}^{R}(S)$, repsectively. Define a germ of analytic mappings of $\mathscr{y}^{R}(S)+\mathscr{H}^{R}(S)$ into $\mathscr{H}^{R}(S)$ by the formula:

$$
\mathbf{G}=\mathbf{F}^{\prime} \circ \mathbf{M} \circ(\mathbf{F}+\mathbf{F})
$$

where $\mathbf{M}$ is the multiplication in $G I L(n)$. It is clear that $\mathbf{G}$ is an infinite Lie $(F)$-group and $\mathbf{F}$ is a representation of $\mathbf{G}$ into $G I L(n)$. By the condition of parameterization mapping, it follows immediately that the cooridnate system $(x), \mathbf{F}$, and $\mathbf{F}^{\prime}$ form a realization of $\mathbf{G}$ as $((\xi), p)$. This finishes the proof of 
Theorem IV. 3.

By a vector field element $\mathbf{f}$ in a manifold $M$, we mean a vector field defined on a non-empty open set $U(\mathbf{f})$ in $M$. Let $\xi$ be a function defined on a neighborhood of $x$. Denote by $\mathbf{f}_{x} \cdot \xi$ the derivative of $\xi$ to the direction of the vector $\mathbf{f}_{x}$ attached at $x$ by $\mathbf{f}$. Denote by $f \cdot \xi$ the function $f_{x} \cdot \xi$ where $x$ is the variable. Let $f^{t}$ be a family of homeomorphism elements of $M$ on an open set $U$ with the parameter space $-\varepsilon<t<\varepsilon$. Assume that $f^{t}$ is the identity mapping of $U$ when $t=0$. Then we can define a vector field element $\mathbf{f}$ on $U$ such that for any $x$ in $U$ and for any function $\xi$ on a neighborhood of $x, f_{x} \cdot \xi$ $=\left(\frac{d}{d t}\right) \xi\left(f^{t}(x)\right)_{t=0}$. $\mathbf{f}$ is called the vector field element defined by the family $f^{t}$, and will be denoted by $\left(\partial f^{t} / \partial t\right)_{t=0}$. For any vector field element $\mathbf{f}$ and for any $x$ in $U(\mathbf{f})$, it is known that there is an open neighborhood $U$ of $x$ and there is a family $f^{t}$ of homeomorphism elements of $M$ on $U$ such that the restriction of $\mathbf{f}$ to $U$ is equal to $\left(\partial f^{t} / \partial t\right)_{t=0}$. Let $\mathbf{g}$ be a vector field element in $M$. If $U(\mathbf{f}) \cap U(\mathbf{g})$ is not empty, we say that the commutator of $\mathbf{f}$ and $\mathbf{g}$ is defined, and the commutator [f, $\mathbf{g}]$ is the vector field on $U(f) \cap U(g)$ such that

$$
[f, \mathrm{~g}]_{x} \cdot \xi=f_{x}(g \cdot \xi)-g_{x}(f \cdot \xi)
$$

for any function $\xi$ defined on a neighborhood of $x$.

Let $\mathbf{f}$ be a vector field element in $M$. We define a vector field element $p^{l}(\mathbf{f})$ in $J^{l}(M)$ such that its domain is $\alpha^{-1}(U(\mathbf{f}))$ as follows: For any pint $x$ in $U(\mathbf{f})$ take a one-parameter family of homeomorphism elements $f^{t}$ such that $\left(\partial f^{t} / \partial t\right)_{t=0}$ is equal to $\mathbf{f}$ on a neighborhood of $x$, then $p^{l}(\mathbf{f})$ is equal to $\left(\partial\left(p^{l}\left(f^{t}\right)\right) / \partial t\right)_{t=0}$ on a neighborhood of $\alpha^{-1}(x)$. If the commutator of $\mathbf{f}$ and $\mathbf{g}$ is defined, so is the commutator of $p^{l}(\mathbf{f})$ and $p^{l}(\mathrm{~g})$ and

$$
p^{l}([\mathbf{f}, \mathbf{g}])=\left[p^{l}(\mathbf{f}), p^{l}(\mathbf{g})\right] .
$$

A collection $\mathfrak{Q}$ of vector field elements in $M$ is called a Lie pseudo-algebra in $M$ if it satisfies the following conditions: If $\mathbf{f}$ and $\mathbf{g}$ is in $\mathfrak{L}$ and if $U(\mathbf{f})$ $\cap U(\mathbf{g})$ is not empty then $[\mathbf{f}, \mathbf{g}]$ and $\lambda \mathbf{f}+\mu \mathbf{g}$ are in $\mathscr{L}$, where $\lambda, \mu$ are real numbers. Let $\mathbb{S}$ be a pseudo-group in $M$. A vector field element $\mathbf{f}$ in $M$ is said to belong to $\$ S$ when for any point $x$ in $U(\mathbf{f})$ there is a neighborhood $U$ of $x$ and there is a one-parameter family $f^{t}$ on $U$ in $\&$ such that $\mathbf{f}$ and $\left(\partial f^{t} / \partial t\right)_{t=0}$ coincide on a neighborhood of $x$. The set $\&$ of vector field ele- 
ments in $M$ which belong to $G$ is a Lie pseudo-algebra in $M$. $?$ will be called the Lie pseudo-algebra associated with $\$$.

A pair of a Lie pseudo-algebra in $M$ and a point in $M$ is called a local Lie pseudo-algebra in $M$. As in the case of pseudo-group we introduce the notion of parameter algebras of local Lie pseudo-algebras. Let $L$ be a formal Lie $(F)$ algebra over the field $\mathbf{R}$ of real numbers. We say that $L$ is an infinite Lie $(F)$-algebra, when the parameter space of $L$ is $H^{I}(S)$ for a system of characters $S$ and when $L$ is the formalization of a germ of analytic mappings of $U^{R}(S)$ $+\mathscr{H}^{R}(S)$. By an infinite Lie $(F)$-algebra, we often mean also the germ of analytic mappings determined by the formal mapping $L$, and so often use the bold letter $\mathrm{L}$ instead of $L$. The formal Lie $(F)$-algebra associated with GIL( :: is an infinite Lie $(F)$-algebra, which will be denoted also by $\operatorname{GIL}(n)$. An in finite Lie $(F)$-algebra $\mathbf{L}$ is called a parameter Lie algebra of a local Lie pseudoalgebra $(\mathfrak{R}, \mathfrak{p})$ in a manifold $M$, if there is a coordinate system $U=\left\{\left(x_{1}, \ldots\right.\right.$, $\left.\left.x_{n}\right) ;\left|x_{j}\right|<\varepsilon\right\}$ in $M$ with center $p$ satisfying the following conditions :

$\left(1^{\circ}\right)$ There is a germ of analytic mappings $\mathbf{F}$ of the parameter space $\mathscr{H}^{n}(S)$ of $L$ into $\mathscr{H}_{n}^{n R}$ scuh that $\mathbf{F}$ is a homorphism of $\mathbf{L}$ into $G I L(n)$; there is a germ of analytic mappings $\mathbf{F}^{\prime \prime}$ of $y_{n}^{n n^{n}}$ into $\mathscr{Y}^{n}(S)$ such that $\mathbf{F}^{\prime} \circ \mathbf{F}$ is the identity mapping of $\mathscr{H}^{R}(S)$.

$\left(2^{\circ}\right)$ There are strictly positive real numbers $u_{1}$ and $a$, and an integer $k$ such that $\mathbf{F}$ is defined on $\mathscr{T}^{I}\left(S ; u, u^{k} a\right)$ for any $u \leq u_{1}$ and such that $\mathbf{F}$ satisfies the following conditions: If $\xi$ is in $y^{R}\left(S ; u, u^{k} a\right)$ for a real number $\boldsymbol{u} \leq \boldsymbol{u}_{1}$ then the vector field $\mathbf{F}_{j}(\xi)\left(\partial / \partial x_{j}\right)$ is in $\mathfrak{I}^{-}$.

$\left(3^{\circ}\right)$ There are strictly positive real numbers $u_{1}^{\prime}$ and $a^{\prime}$ and an integer $k^{\prime}$ with the following conditions: If $\eta$ is in $\mathscr{H}_{n}^{n F}\left(u, u^{h^{\prime}} a^{\prime}\right)$ for a real number $u \leq u_{1}^{\prime}$ and if the vector field $\eta_{i}\left(\partial / \partial x_{i}\right)$ is in $\mathbb{\Omega}^{-}$then $\mathbf{F}\left(\mathbf{F}^{\prime}(\eta)\right)=\eta$. The collection of $U, \mathbf{F}$, and of $\mathbf{F}^{\prime}$ such as above will be called a realization of $\mathbf{L}$ as $(\Omega, p)$. The following theorem is easy to prove:

THEOREM IV.4. Let $(\mathfrak{G}, \mathfrak{p})$ be a local continuous pseudo-group in a manifold $M$. Take a parameter group $\mathrm{G}$ of $(\mathfrak{G}, \mathfrak{b})$. Let a collection $\left\{U, \mathbf{F}, \mathbf{F}^{\prime}\right\}$ be $a$ realization of $\mathbf{G}$ as $(\mathfrak{G}, \mathfrak{p})$. Denote by $\&$ the Lie psendo-algebra in Massociated with (\$S. Let $\mathbf{L}$ be infinite Lie (F)-algebra associated with $\mathbf{G}$. Then $\mathbf{L}$ is a parameter algebra of $(\mathcal{Q}, n)$ and the collection $\left\{U,(d \mathbf{F})_{0},\left(d \mathbf{F}^{\prime}\right)_{n}\right\}$ is a realization

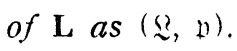




\section{$\S 2$. Structure equations}

Let $D$ be a domain $\left\{\left(x_{1}, \ldots, x_{n}\right) ;\left|x_{j}\right|<\varepsilon\right\}$. Then $G^{l}(D)$ is a domain in a space $\left(x_{1}, \ldots, x_{n}, y_{1}, \ldots, y_{n}, p\right)$, where $p=\left(\ldots, p_{j}^{j_{1} \ldots j_{\nu}}, \ldots\right)(\nu \leq l$, $p_{j}^{j_{1} \cdots j_{\nu}}$ is symmetric with respect to permutations of indices $\left.j_{1}, \ldots, j_{\nu}\right)$, by setting $x=\alpha(X), y=\beta(X)$, and

$$
p_{j}^{j_{1} \cdots j_{\nu}}=\partial^{\nu} f_{i} / \partial x_{j_{1}} \cdots \partial x_{j_{\nu}}
$$

where $X=j_{x}^{l}(f)$ and $f\left(x^{\prime}\right)=\left(f_{1}\left(x^{\prime}\right), \ldots, f_{n}\left(x^{\prime}\right)\right)$. Let $e_{j}(x)$ and $e_{j}^{j_{j} \ldots j_{\nu}}(x)$ be the linear functionals $d x_{j}$ and $d p_{j}^{j_{1} \cdots j_{\nu}}$ on the tangent vector space to $G^{l}(D)$ at $I^{\prime}(x)$, respectively. Take $X=j_{x}^{l}(f)$ in $G^{l}(D)$. Then by the definition $\left[p^{l}(f)\right](X)$ $=I^{l}(y)$, where $y=\beta(X)$. Hence $p^{l}(f)$ induces a linear mapping $p(f)^{*}$ of the conjugate space of the tangent vector space to $G^{l}(D)$ at $I^{l}(y)$ to the conjugate space of the tangent vector space to $G^{l}(D)$ at $X$. Set $\left(\varphi_{j}\right)_{X}=p^{l}(f)^{*}\left(e_{j}(y)\right)$, $\left(\varphi_{j}^{j_{1} \cdots j_{\nu}}\right)_{X}=p^{l}(f)^{*}\left(e_{j}^{j_{1} \cdots j_{\nu}}(y)\right)$. It is clear that $\left(\varphi_{i}\right)_{X}$ and $\left(\varphi_{j}^{j_{1} \cdots j_{\nu}}\right)_{X}$ do not depend upon the choice of $f$ such that $X=j_{x}^{\prime}(f)$, provided $\nu \leq l-1$. It is also clear that there are (real analytic) differential forms ${ }^{l} \varphi_{j}$ and ${ }^{l} \varphi_{j}^{j_{1} \cdots j_{\nu}}\left({ }_{\nu} \leq l-1\right)$ on $G^{l}(D)$ such that linear functionals of the tangent vector space at $X$ assigned by ${ }^{l} \varphi_{j}$ and ${ }^{l} \varphi_{j}^{j_{j} \cdots j_{v}}$ are equal to $\left(\varphi_{j}\right)_{X}$ and $\left(\varphi_{j}^{j_{1} \cdots j_{\nu}}\right)_{X}$, respectively. By the defini-

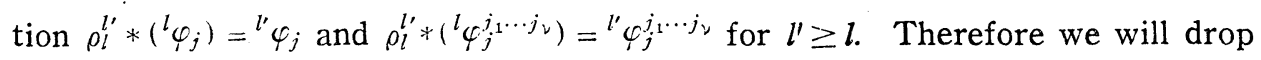
index $l$ and write $\varphi_{j}, \varphi_{i}^{j_{1} \cdots j_{v}}$ (instead of ${ }^{l} \varphi_{j},{ }^{l} \varphi_{j}^{j_{1} \cdots j_{\nu}}$ ) as far as no confusion can occur. Also we will consider $\varphi_{j}$ as the case $\nu=0$ of $\varphi_{j}^{j_{1} \cdots j_{2}}$.

Let $(\mathbb{S}, \mathfrak{p})$ be a local continuous pseudo-group in a manifold $M$. Let $(M$, $\left.M^{\prime}, \pi\right)$ be the fibered manifold of invariants of $(B)$ Take a coordinate system $U=\left\{\left(x_{1}, \ldots, x_{n}\right) ;\left|x_{j}\right|<\varepsilon\right\}$ in $M$ with center $p$ such that $\left(x_{1}, \ldots, x_{h}\right)$ can be considered as a coordinate system in $M^{\prime}$ with center $\pi(p)$ for an integer $h(0 \leq h \leq n)$. Set $A^{l}(\mathbb{B} \mid U)=A^{l}(\mathbb{B}) \cap G^{l}(U)$. For $f$ in $\mathbb{S}^{-}, p^{l}(f)$ preserves $A^{l}(\leftrightarrow)$. We will denote by the same letter $p^{l}(f)$ its restriction to $A^{l}(\& \mid U)$.

Proposition IV.4. Let $h$ be a function defined on an open set $V$ in $A^{l}(\$)$. If $h \cdot p^{l}(f)=h$ for any $f$ in $\left(B\right.$, there is a function $h^{\prime}$ defined on $\beta(V)$ such that $h=h^{\prime} \circ \beta$. If moreover $V$ is in $A^{l}(\mathbb{S} \mid U)$, then $h$ is a function of $x_{1} \circ \alpha, \ldots, x_{i} \circ \alpha$, $x_{h+1} \circ \beta, \ldots, x_{n} \circ \beta$.

Proof. For $X$ in $A^{l}((\$))$, take $f$ in $\&$ such that $X=j_{x}^{l}(f)$. Then $\left[p^{l}(f)\right](X)$ $=I^{l}\left(\beta\left(X^{*}\right)\right)$. Therefore $p^{l}(\Theta)$ is a pseudo-group of fiber preserving transformations of $\left(A^{l}(\mathbb{B}), M, \beta\right)$, which induces the identity mapping on $M$ and which 
is transitive on the fibers. Hence there is a function $h^{\prime}$ on $\beta(V)$ such that $h=h^{\prime} \circ \beta$. The last assertion clearly holds because $x_{1} \circ \beta=x_{1} \circ \alpha, \ldots, x_{h} \circ \beta$ $=x_{h} \circ \alpha$ on $\left.A^{l}(\$) \mid U\right)$.

We identify the open set $U=\left\{(x) ;\left|x_{j}\right|<\varepsilon\right\}$ with a domain $D$ in $\mathbf{R}^{n}$. Thus the differential forms $\varphi_{j}^{j_{1} \cdots j_{\nu}}$ defined in the beginning of this section is defferential forms on $G^{l}(U) \subseteq G^{l}(M)$. Denote by ${ }^{\prime} \omega_{j}^{j_{1} \cdots j_{\nu}}$ the restriction of $\varphi_{j}^{j_{1} \cdots j_{\nu}}$ on $A^{l}(\& \mid U)$.

Proposition IV.5. Let, $\omega$ be a Pfaffian form on an open set $V$ in $A^{l}(\$ \mid U)$. Assume that $p^{l}(f) *{ }^{\prime} \omega={ }^{\prime} \omega$ for any $f$ in $\$$. Then locally we have ' $\omega$ $=\left(a_{j_{1} \ldots j_{\nu} \circ \beta}^{j}\right)^{\prime} \omega_{j}^{j_{j} \cdots j_{\nu}}+\beta^{*} \psi(\nu \leq l-1)$, where $a_{j_{1} \ldots j_{2}}^{j}$ and $\psi$ are functions and $a$ differential form defined on $\beta(V)$, respectively.

Proof. Take $q_{1}, \ldots, q_{m_{l}}$ among $p_{j}^{j_{1} \cdots j_{l}}$ such that $d q_{\lambda}$ form a base of 1 -forms on each fiber $\left(A^{l}(\mathbb{S} \mid U), A^{l-1}(\mathbb{S} \mid U), \rho_{l-1}^{l}\right)$. We can write

$$
\left({ }^{\prime} \omega\right)_{l^{\prime}(y)}=a^{j}(y) d x_{j}+a_{j_{1} \ldots j_{\sigma}}^{j}(y) d p_{j}^{j_{1} \cdots j_{\sigma}}+b^{j}(y) d y_{j}+c^{\lambda}(y) d q_{\lambda}, \quad(1 \leq \sigma \leq l-1) .
$$

Therefore by the definition of ${ }^{\prime} \omega_{j}^{j_{1} \cdots j_{\nu}}$ we have

$$
\left({ }^{\prime} \omega\right)_{X}=a^{j}(\beta(X))^{\prime} \omega_{j}+a_{j}^{j_{1} \cdots j_{\sigma}}(\beta(X))^{\prime} \omega_{j}^{j_{j} \cdots j_{\sigma}}+b^{j}(\beta(X)) d y_{j}+\xi,
$$

where $\xi$ is a differential form such that $(\xi)_{l l_{(y)}}=c^{\lambda}(y) d q_{\lambda}$. On the other hand, for any $X$ and $X^{\prime}$ in $A^{l}(\mathbb{B})$ with $\rho_{l-1}^{l}(X)=\rho_{l-1}^{l}\left(X^{\prime}\right)$, there is $f$ in $\&$ such that $\left[p^{l}(f)\right](X)=X^{\prime}$ and such that $\rho_{l-1}^{l}\left[p^{l}(f)\right]\left(X^{\prime \prime}\right)=\rho_{l-1}^{l}\left(X^{\prime \prime}\right)$ for any $X^{\prime \prime}$ in $A^{l}(\mathbb{S})$ with $(\alpha \times \beta)\left(X^{\prime \prime}\right)=(\alpha \times \beta)(X)$. Therefore $(\xi)_{l l(y)}=0$. Since $\xi$ is invariant under $p^{l}(B), \xi$ is zero. This finishes the proof.

Denote by $\rho_{1}$ and $\rho_{2}$ the projection of $G^{l}(D) \times G^{l}(D)$ to the first and the second factors, respectively. Let $\Sigma_{D}$ be the differential system on $G^{l}(D) \times G^{l}(D)$ generated by

$$
\Sigma_{D}: \beta_{j} \circ \rho_{2}-\beta_{j}^{\circ} \rho_{1}, \quad \rho_{2}^{*} \varphi_{j}^{j_{1} \cdots j_{\nu}}-\rho_{1}^{*} \varphi_{j}^{j_{1} \cdots j_{\nu}}, \quad(0 \leq \nu \leq l-1),
$$

and their exterior derivatives. Now take a cross-section $R$ of the fibered manifold $\left(G^{l}(D), D, \alpha\right)$. Denote by $\kappa_{R}$ the mapping of $G^{l}(D)$ into $G^{l}(D) \times G^{l}(D)$ defined by

$$
\kappa_{R}(X)=\left(R(\alpha(X)), R(\alpha(X)) \circ X^{-1}\right) .
$$

$\kappa_{R}$ is an injection of the manifold $G^{l}(D)$ and the image of $\kappa_{R}$ is the submanifold $\left\{(R(x), Y) ; x \in D, Y \in G^{l}(D), \beta(Y)=\beta(R(x))\right\}$. For any homeomorphism. 
element $h$ of $D, j^{l}(h)$ is a local cross-section of $\left(G^{l}(D), D, \alpha\right)$. Since $\kappa_{R}\left(j^{l}(h)\right)$ is a submanifold of $G^{l}(D) \times G^{l}(D)$ expressed parametrically by $(R(x)$, $\left.R(x) \circ j_{x}^{l}(\dot{h})^{-1}\right)=\left(R(x),\left[p^{l}(h)\right](R(x))\right)$ and since $p^{l}(h) * \varphi_{j}^{j_{1} \cdots j_{\nu}}=\varphi_{j}^{j_{1} \cdots j_{\nu}}$, it follows that $j^{l}(h)$ is an integral of $\kappa_{R}^{*}\left(\Sigma_{D}\right)$. On the other hand, $G^{l}(D)$ is canonically identified with with an open submanifold of $f^{l}\left(J^{\circ}(D), D, \alpha\right)$ (cf. Chapt. III. 1$)$. Then by the definition of $\Pi\left(l ;\left(J^{\circ}(D), D, \alpha\right)\right)$ it follows that $\kappa_{R}^{*}\left(\Sigma_{D}\right)$ is contained in $I\left(l ;\left(J^{\circ}(D), D, \alpha\right)\right)$ (cf. the paragraph preceeding Prop. III.1). By counting the number of linearly independent elements, we find that

$$
\kappa_{R}^{*}\left(\Sigma_{D}\right)=\Pi\left(l ;\left(J^{\circ}(D), D, \alpha\right)\right) .
$$

Proposition IV.6. There is an integer $l_{1}$ such that for any $l \geq l_{1}$ the following holds: Let $F$ be a homeomorphism element of $A^{l}(\$ \mid U)$ and let $X^{\circ}$ be in $U(F)$. Then the following two conditions are equivalent:

$\left(1^{\circ}\right)$ there is a homeomorphism element $f$ in $\mathbb{B}^{-}$defined on a neighborhood of $x^{\circ}=\alpha\left(X^{\circ}\right)$ such that $F$ and $p^{l}(f)$ coincide on a neighborhood of $X^{\circ}$ in $A^{l}(\Theta)$,

$\left(2^{\circ}\right) \quad F *^{\prime} \omega_{j}^{j_{1} \cdots j_{\nu}}={ }^{\prime} \omega_{j}^{j_{1} \cdots j_{\nu}} \quad(0 \leq \nu \leq l-1), \quad \beta \circ F=\beta$

on a neighborhood of $X^{\circ}$.

Proof. By the definition of ${ }^{\prime} \omega_{j}^{j_{1} \cdots j_{\nu}}$, it is clear that $\left(1^{\circ}\right)$ implies $\left(2^{\circ}\right)$. As sume conversely that $\left(2^{\circ}\right)$ holds. Take $g$ in $\&$ such that $X^{\circ}=j_{3\left(X^{\circ}\right)}^{l}(g)^{-1}$. Considering $F \circ p^{l}(g)$ instead of $F$, we can assume without loss of generality that $X=I^{l}\left(x^{\circ}\right) . \quad \varphi_{1}, \ldots, \varphi_{n}$ are linear combinations of $d x_{1}, \ldots, d x_{n}$ with a nonsingular coefficient matrix, and so are ' $\omega_{1}, \ldots,{ }^{\prime} \omega_{n}$. Hence there is a neighborhood $\mathscr{U}$ of $I^{l}\left(x^{\circ}\right)$ such that for any $X$ in $\mathscr{U}$

$$
F(X)=h(\alpha(X))
$$

where $h$ is a mapping into $U$ defined on a neighborhood of $x^{\circ}$.

$\Sigma_{D}$ defined by the formula (2) can be considered as a differential system on $G^{l}(U) \times G^{l}(U)$. Denote by $\Sigma_{\mathscr{S}}$ the restriction of $\Sigma_{D}$ to $A^{l}(\Theta \mid U) \times A^{l}(\Theta \mid U)$. Denote by $N$ the submanifold of $A^{l}(\mathscr{B} \mid U) \times A^{l}(\mathscr{S} \mid U)$ expressed parametrically by $(X, F(X)),(X \in \mathscr{U})$. Since $F$ satisfies $\left(2^{\circ}\right), N$ is an integral of $\Sigma_{\mathscr{G}}$. Let $R$ be a local cross-section of $\left(A^{l}(\Im \mid U), U, \alpha\right)$. Then $R$ is at the same time a local cross-section of $\left(G^{\prime}(U), U, \alpha\right)$. Denote by the same letter the restriction of $\kappa_{R}$ (defined by the formula $\left.(2)\right)$ to $A^{l}(B \mid U)$. Then the local cross-section which send $x$ to $\left(R(x)^{-1} \circ F(R(x))\right)^{-1}$ of $\left(A^{l}((\mathscr{G} \mid U), U, \alpha)\right.$ is mapped by $\kappa_{R}$ into 
$N$. Therefore the local cross-section defined above is an integral of $\kappa_{R}^{*}\left(\Sigma_{\mathbb{S}}\right)$. On the other hand, $\kappa_{R}^{*}\left(\Sigma_{\mathscr{S}}\right)$ is the restriction of $\Pi\left(l ;\left(J^{\circ}(U), U, \alpha\right)\right)$ to $A^{l}(\& \mid U)$ (cf (4)). Hence by Prop. III. 2 , there is a homeomorphism element $f$ of $U$ defined on a neighborhood of $x^{\circ}$ such that

$$
R(x)^{-1} \circ F(R(x))=j_{x}^{l}(f)^{-1} .
$$

Therefore $F(R(x))=R(x) \circ j_{x}^{l}(f)^{-1}$. By appling $\alpha$ on the both sides of this equality, we find by $(5)$ that $f(x)=h(x)$. Thus $F$ is equal to $p^{l}(h)$ on any local cross-section of $\left(A^{l}(\mathbb{S} \mid U), U, \alpha\right)$. Hence $F=p^{l}(h)$. Now take $l$ so large that the condition $\left(1^{\circ}\right)$ of Thereom IV. 1 holds for $l$. Then since $j_{x}^{l}(h)$ $=F\left(I^{l}(x)\right)^{-1} \in A^{l}(\mathbb{S}), h$ is in $\mathbb{S}^{-}$. Thus we finish the proof.

Let $\psi_{j}^{j_{1} \cdots j_{\nu}}(1 \leq \nu \leq l)$ be the invariant Pfaffian form on $G_{x}^{l}(U)$ such that $\left(\phi_{j}^{j_{1} \cdots j_{\nu}}\right)_{L^{l}(x)}=\left(d p_{j}^{j_{1} \cdots j_{\nu}}\right)_{I^{l}(x)}$. Then

$$
d \psi_{j}^{j_{1} \cdots j_{\nu}}=\frac{1}{2} c_{j}^{j_{1} \cdots j_{\nu} i i_{1} \cdots i_{\sigma} k^{k} \cdots k \tau} \psi_{i_{1} \cdots i_{\sigma}}^{i} \wedge \psi_{k}^{k_{1} \cdots k \tau}
$$

where $c$ 's are constants and independent of $x$, anti-symmetric with respect to the exchange of $\left(i ; i_{1}, \ldots, i_{s}\right)$ and $\left(k ; k_{1}, \ldots, k_{\tau}\right)$. Moreover they are independent of $l$, provided $l \geq \nu, \sigma, \tau$.

Lemma IV.2. We have on $G^{l}(U)$ the following equalities:

$$
\begin{aligned}
& d \varphi_{j}=\varphi_{j}^{k} \wedge \varphi_{k} \\
& d \varphi_{i}^{j_{1} \cdots j_{\nu}}=\frac{1}{2}-c_{j}^{j_{1} \ldots j_{\nu}} \underset{i_{1} \ldots i_{\sigma} k_{1} \ldots k \tau}{k} \varphi_{i}^{i_{1} \cdots i_{\sigma}} \wedge \varphi_{k}^{k_{1} \cdots k \tau}+\varphi_{j}^{j_{1} \cdots j_{\nu} k} \wedge \varphi_{k} \\
&(1 \leq \nu, \sigma, \tau \leq l-2)
\end{aligned}
$$

Proof. By the construction, the restriction of $\varphi_{j}^{j_{1} \cdots j_{\nu}}$ to $\mathbb{S}_{x}^{l}$ is equal to $\psi_{j}^{j_{1} \cdots j_{\nu}}$. On the other hand, when we employ the coordinate system $(x, y, p), \varphi_{j}^{j_{1} \cdots j_{v}}$ can be expressed as a differential form of $(x, p)$. Therefore we have

$$
d \varphi_{j}^{j_{1} \cdots j_{\nu}}=\frac{1}{2} c_{j}^{j_{j} \cdots j_{\nu} i} \underset{i_{1} \cdots i_{\sigma} k_{1} \cdots k \tau}{k} \varphi_{i}^{i_{1} \cdots i_{\sigma}} \wedge \varphi_{k}^{k_{1} \cdots k \tau}+a_{j}^{j_{1} \cdots j_{\nu}{ }_{i_{1} \cdots i_{\sigma} \sigma}^{i}}{ }^{k} \varphi_{i}^{i_{1} \cdots i_{\sigma}} \wedge \varphi_{k}
$$

Since $\varphi^{\prime}$ 's are invariant under $p^{l}(f)$ for any homeomoephism element $f$ of $U$, we can choose the above expression so that a's are constants. Hence it is sufficient to find a's at $I^{l}(x)$. This can be done by direct calculation.

We remark that, under the coordinate $\operatorname{system}(x, y, p), \varphi_{j}^{j_{1} \ldots j_{\nu}}$ are linear combinations of $d x_{1}, \ldots d x_{n}, \ldots, d p_{k}^{k_{1} \ldots k \tau}, \ldots$ with $\tau \leq \nu$ and are linearly 
independent on $G^{l}(U)$. Therefore we can choose, for each $l$, ' $\omega_{n_{l-2}+1}, \ldots$, ' $\omega_{n_{l-1}}$ among ' $\omega_{j}^{j_{1} \cdots j_{l-1}}$ on $A^{l}(\mathbb{B} \mid U)$ such that ${ }^{\prime} \omega_{1}, \ldots,{ }^{\prime} \omega_{n_{l-1}}$ at $I^{l}(\mathfrak{p})$ are linearly in. dependent. Take a mapping $\tau$ of a neighborhood ' $\mathscr{U}^{l}$ of $I^{l}(p)$ in $A^{l}(\& \mid U)$ into $A^{l+1}(\Theta \mid U)$ such that $\rho_{l}^{l+1} \circ \tau$ is the identity mapping. Set ' $\omega_{\lambda}^{l}=\tau^{* \prime} \omega_{n_{l-1}+\lambda}$. Take $\mathscr{U}^{l}$ in ' $\mathscr{U}^{l}$ such that ' $\omega_{1}, \ldots, \iota_{n_{l-1}},{ }^{\prime} \omega_{1}^{l}, \ldots,{ }^{\prime} \omega_{n_{l}}^{l}, \quad\left(m_{l}=n_{l}-n_{l-1}\right), d y_{j_{1}}, \ldots$, $d y_{j_{a}}$ form a base of Pfaffian forms on $\mathscr{U}^{l}$. Then we have for $\nu \leq l-1$

$$
{ }^{\prime} \omega_{j}^{j_{1} \cdots j_{\nu}}=a_{j}^{j_{1} \cdots j_{\nu} \mid k,} \omega_{k} \quad\left(k=1, \ldots, n_{\nu}\right)
$$

on $\mathscr{U}^{l}$, where $a_{j}^{j_{1} \cdots j_{\vee} \mid k}$ is an invarinat function of $p^{l}(\&)$. Considering the above equality for $\nu=l+1$ on $\mathscr{U}^{l+1}$, we find that

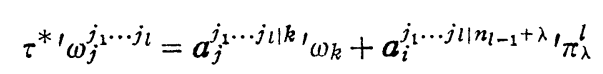

Hence by Lemma IV. 2

$$
d^{\prime} \omega_{i}=\frac{1}{2} c_{i}^{i k \prime} \omega_{j} \wedge^{\prime} \omega_{k}+a_{i}^{j \lambda \prime} \omega_{j} \wedge^{\prime} \pi_{\lambda}^{l} \quad\left(c_{i}^{j k}+c_{i}^{k j}=0, i, j, k=1, \ldots, n_{l-1}\right)
$$

where $c_{i}^{j k}$ and $a_{i}^{j \lambda}$ are invariant functions of $p^{l}(\mathbb{S})$.

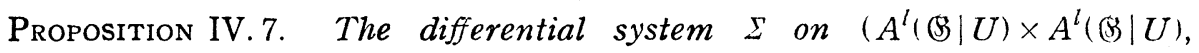
$\left.A^{l}(\mathbb{S} \mid U), \rho_{1}\right)$ generated by $\rho_{1}^{*} \omega_{j}^{j_{1} \cdots j_{\nu}}-\rho_{2}^{*}{ }^{\prime} \omega_{j}^{j_{1} \cdots j_{\nu}}(0 \leq \nu \leq l-1), \beta_{i} \circ \rho_{1}-\beta_{j} \circ \rho_{2}$, and their exterior derivatives is in involution at every integral points for any suffciently large $l$.

Proof. (i) Denote by $Q^{r}$ the manifold of $r$-dimensional contact elements $E^{r}$ to $A^{l} \times A^{l}$, where $A^{l}=A^{l}(\& \mid U)$, such that $d \rho_{1} E^{r}$ is still $r$-dimensional. Let $\mathscr{I} Q^{r}$ be the set of integral elements in $Q^{r}$.

(i.1) The case $r \leq n$. Let $E^{r}$ be in $\mathscr{B} Q^{r}$. Assume that there is a local cross-section $R$ of $\left(A^{l}, U, \alpha\right)$ such that $E^{r}$ is tangent to the submanifold $N_{R}$ $=\{(R(x), Y) ; \beta(Y)=\beta(R(x))\}$ in $A^{l} \times A^{l}$. Then $d \kappa_{R}^{-1} E^{r}$ is an integral element of $\kappa_{R}^{*}(\Sigma)(\operatorname{cf}(3))$. By (4) and Theorem IV.1, $\kappa_{R}^{*}(\Sigma)$ is in involution for sufficiently large $l$. Assume further that $d \kappa_{R}^{-1} E^{r}$ is an ordinary integral element of $\kappa_{R}^{*}(\Sigma)$. Then there is an integral $f$, i.e., $f$ in $\mathbb{B}$, of $\kappa_{R}^{*}(\Sigma)$ such that $d \kappa_{R}^{-1} E^{r}$ is tangent to the integral manifold $j^{l}(f)$. Then $E^{r}$ is tangent to the graph of $p^{l}(f)$, which is an integral of $\Sigma$. Thus the rank of polar equation of $E^{r}$ is equal to the reduced polar eqution of $E^{r}$. Denote by $\left(X^{\circ}, Y^{\circ}\right)$ the foot point of $E^{r}$. Then the tangent vector space to $A^{l} \times A^{l}$ at $\left(X^{\circ}, Y^{\circ}\right)$ is spuned by that of $N_{R}$ at $\left(X^{\circ}, Y^{\circ}\right)$ and tangent vectors of the form $(L, L)$ where $L$ is an 
tangent vector to $A^{l}$. By Lemma IV.2, $\Sigma$ is generated as an ideal by elements of the forms : $\rho_{1}^{*} \theta-\rho_{2}^{*} \theta$ (where $\theta$ being 1-forms or functions) and linear combination of $\rho_{1}^{*}{ }^{\prime \prime} \omega_{j}^{j_{1} \cdots j_{\nu}} \wedge\left(\rho_{1}^{*} \theta-\rho_{2}^{*} \theta\right)$ (where $\theta$ being 1 -forms). Then it is easy to check that the rank of reduced polar equation of $E^{r}$ is equal to its restriction to the tangent vector space to $N_{R}$, because any tangent vector of the form $(L, L)$ is a solution of the reduced polar equation of $E^{r}$. Hence $t\left(E^{r}\right)=t_{r}^{*}$ where $t_{r}^{*}$ is the rank of the polar equation of the generic integral element of dimension $r$ of the exterior differential system associated with $\left(\mathscr{D}^{l}(\mathbb{B}),(M \times M\right.$, $\left.M, \rho_{1}\right)$ ) (cf. Theorem IV. 1).

(i.2) The case $r \geq n$. Let $E^{r}$ be in $\mathscr{B} Q^{r}$. Assume that $E^{r}$ contains $E^{r}$ which satisfies the regularity conditions in (i.1). Then by the similar argument as in (i.1) it is easy to check that $t\left(E^{r}\right)=t_{r}^{*}$.

(ii) Let $E$ be an $N$-dimensional integral element, $N=\operatorname{dim} A^{l}$, in $Q^{N}$. We have to show that there is $E^{r} \subseteq E$ with $E^{r} \in Q^{r}$ such that $t$ is a constant function on a neighborhood of $E^{r}$ in $\mathscr{I} Q^{r}$. We can always find $E^{r} \subseteq E$ with $E^{r} \in Q^{r}$ which satisfies the regularity conditions in (i). Since any integral element sufficiently near to $E$ also satisfies the same regularity conditions, it is clear that the function $t$ is the constant $t_{r^{\prime}}^{*}, r^{\prime}=\operatorname{Min}(r, n)$, on a neighborhood of $E^{\prime \prime}$ in $\mathscr{G} Q^{r}$. Thus we proved that $\Sigma$ is in involution at any integral points.

Let $\tau$ be a local cross-section of $\left(M, M^{\prime}, \pi\right)$ defined on a neighborhood of

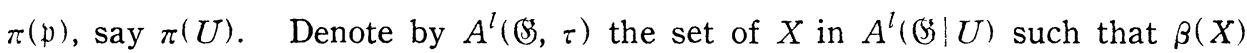
$=\tau(\pi \alpha(X))$. For any $f$ in $\mathbb{S}^{-}$such that $U(f) \cap V(f) \subseteq U, p^{l}(f)$ preserves the submanifold $A^{l}(\oiint, \tau)$. Denote by $p^{l}(\$, \tau)$ the set of homeomorphism elements $F$ in $A^{l}(\mathfrak{S}, \tau)$ with the following conditions: For any $X$ in $U(F)$ there is $f$ in (S) such that $F$ and $p^{l}(f)$ coincide on a neighborhood of $X$ in $U(F)$. Clearly $p^{l}(\mathbb{S}, \tau)$ is a pseudo-group in $A^{l}(\mathbb{S}, \tau)$. Denote by $\omega_{j}^{j_{1} \cdots j_{v}}$ the restriction of ${ }^{\prime}\left(\omega_{j}^{j_{1} \cdots j_{\gamma}}\right.$ to $A^{l}(\mathbb{S}, \tau)$. We denote by the same letter $\alpha$ the restriction to $A^{l}(\mathbb{S}, \tau)$ of $\alpha: G^{l}(U) \rightarrow U . \quad\left(A^{l}(\Theta, \tau), U, \alpha\right)$ is a fibered manifold. By exactly the same method we used for $p^{l}((B) U)$ we prove the following:

Proposition IV.8. Let $(\mathfrak{G}, \mathfrak{p})$ be a local continuous pseudo group in $M$. Denote by $\left(M, M^{\prime}, \pi\right)$ the fibered manifold of invariants of $(\mathcal{S}$. Let $U$ be $a$ sufficiently small coordinate neighborhood of $p$. Let $\tau$ be a local crosssection of $\left(M, M^{\prime}, \pi\right)$ defined on $\pi(U)$. Then we have the following:

$\left(1^{\circ}\right) p^{l}(\circlearrowleft, \tau)$ is a continuous pseudo-group in $A^{l}(\mathbb{S}, \tau)$ and $\left(A^{l}(\mathbb{S}, \tau), \pi(U)\right.$, 
$\pi \circ \alpha)$ is the fibered manifold of invariants of $p^{l}(\mathbb{G}, \tau)$.

$\left(2^{\circ}\right)$ Any Pfaffian form $\omega$ defined on an open set in $A^{l}(\mathbb{S}, \tau)$ invariant under any $F$ in $p^{l}(\mathbb{S}, \tau)$ can be expressed as a linear combination of $\omega_{j}^{j_{1} \cdots j_{\nu}}$ in a neighborhood of each point, with coefficients in the ring of invariant functions.

$\left(3^{\circ}\right)$ A homeomorphism element $F$ of $A^{l}(\mathfrak{B}, \tau)$ belongs to $p^{l}(\mathfrak{B}, \tau)$ if and only if $\pi^{\wedge} \alpha \circ F=F$ and $F^{*} \omega_{j}^{j_{1} \cdots j_{\nu}}=\omega_{j}^{j_{1} \cdots j_{\nu}}(0 \leq \nu \leq l-1)$.

$\left(4^{\circ}\right)$ The differential system on $\left(A^{l}(\mathfrak{S}, \tau) \times A^{l}(\mathfrak{S}, \tau), A^{l}(\mathfrak{S}, \tau), \rho_{1}\right)$ generated by $\rho_{1}^{*} \omega_{j}^{j_{1} \cdots j_{\nu}}-\rho_{2}^{*} \omega_{j}^{j_{1} \cdots j_{\nu}}(0 \leq \nu \leq l-1)$ and $\pi^{\circ} \alpha^{\circ} \rho_{1}-\pi^{\circ} \alpha \circ \rho_{2}$ is in involution at every integral point, where $\rho_{1}, \rho_{2}$ is the projection to the first and second factors, respectivly.

Moreover, we have the following:

Theorem IV.5. Under the notations in Prop. IV.8, denote by nl the dimension of $A^{l}(\xi, \tau)$. Then we can find an open neighborhood $\mathscr{U}^{l}$ of $I^{l}(p)$ in $A^{l}(\mathbb{S}, \tau)$ and Pfaffian forms $\omega_{n_{l-2}-1}, \ldots, \omega_{n_{l-1}}, \pi_{1}^{l}, \ldots, \pi_{m_{l}}^{l}\left(m_{l}=n_{l}-n_{l-1}\right)$ on $\mathscr{U}^{l}$ satisfying the following conditions for sufficiently large $l$ :

$\left(^{\circ}\right) \quad \omega_{1}, \ldots, \omega_{n_{l-1}}, \pi_{1}^{l}, \ldots, \pi_{m_{l}}^{l}$ form a base of Pfaffian forms on $t^{l}$ and

$$
\begin{array}{ll}
d \omega_{l}=\frac{1}{2} & c_{i}^{j k} \omega_{j} \wedge \omega_{k}+a_{i}^{j \lambda} \omega_{j} \wedge \omega_{\lambda}^{l} \quad\left(c_{i}^{j k}+c_{i}^{k j}=0\right) \\
a_{i}^{j \wedge}=0 & \left(i \leq n_{l-2}, \text { or } j>n\right)
\end{array}
$$

where $c_{i}^{j k}$ and $a_{i}^{j \lambda}$ are invariant functions of $p^{l}(\dot{G}, \tau)$.

$\left(2^{\circ}\right)$ A homeomorphism element $F$ of is in $p^{l}(\mathbb{B}, \tau)$ if and only if

$$
\pi \circ \alpha \circ F=F, \quad F^{*} \omega_{i}=\omega_{i} \quad\left(i=1, \ldots, n_{l-1}\right)
$$

where $\left(M, M^{\prime}, \pi\right)$ is the fibered manifold of invariants of $(B)$

$\left(3^{\circ}\right)$ Take a coordinate system $\left(w_{1}, \ldots, w_{h}\right)$ of $M^{\prime}$ on $\pi^{\circ} \alpha\left(\mathscr{C}^{l}\right)$. The exterior differential system on $\left(\mathscr{U}^{l} \times \mathscr{C}^{l}, \mathscr{U}^{l}, \rho_{1}\right)$ generated by

$$
\rho_{1}^{*} \omega_{i}-\rho_{2}^{*} \omega_{i}, \quad w_{r} \circ \rho_{1}-w_{r} \circ \rho_{2} \quad\left(i=1, \ldots, n_{l-1} ; r=1, \ldots, h\right)
$$

is in involution at each integral points of $\dddot{y}^{l} \times \mathscr{l}^{l}$, where $\rho_{1}, \rho_{2}$ is the projertion to the first, second factors, respectively.

Assume that $(B)$ is transitive on $M$. Then the base space of the fibered manifold of invariants of $\mathbb{B}$ is a single point. So $\mathfrak{p}$ in $M$ defines a cross-section $\tau_{p}$ of the fibered manifold. We set 


$$
A^{l}\left(\mathbb{B}, \tau_{p}\right)=A^{l}(\mathbb{B}, \mathfrak{p}), \quad p^{l}\left(\mathbb{B}, \tau_{\mathfrak{p}}\right)=p^{l}(\mathbb{B}, \mathfrak{p}) .
$$

In the remainder of this section, we study relations between $c_{i}^{j k}, a_{i}^{j \lambda}$ in Theorem IV.5. and the bracket operation in the parameter Lie $(F)$-algebra of $(\mathfrak{B}, \mathfrak{b})$, in the case $\mathbb{S}$ is transitive. These relations will be used in $\S 5$.

Let $\mathfrak{Z}$ be the Lie pseudo-algebra associated with $\mathbb{G}$. For $\mathbf{f}$ in $\mathfrak{Q}$ and for $X$ in $A^{l}(\mathbb{S}, \tau) \cap U\left(p^{l}(\mathbf{f})\right)$, it is clear that $\left(p^{l}(\mathbf{f})\right)_{x}$ is tangent to $A^{l}(\mathbb{S}, \tau)$. Now, fix $\omega_{i}, \pi_{\lambda}^{l}, \mathscr{U}^{l}$ such as in Theorem IV.5. Take a system of functions $u_{n_{l-2}+1}$, $\ldots, u_{n_{l-1},}, v_{1}^{l}, \ldots, v_{m_{l}}^{\prime}$ on $\mathscr{C}^{l}$ such that $u_{1}, \ldots, u_{n_{l-1}}, v_{1}^{l}, \ldots, v_{m_{l}}^{l}$ is a coordinate system on $\mathscr{U}^{l}$ and such that

$$
\left(\omega_{i}\right)_{l} l_{(\mathfrak{p})}=\left(\partial / \partial u_{i}\right), \quad\left(\pi_{\lambda}^{l}\right)_{l} l_{(\mathfrak{p})}=\left(\partial / \partial v_{\lambda}^{l}\right)
$$

For $\mathbf{f}, \mathbf{g}$ in $\mathfrak{Q}$ such that $U(\mathbf{f}) \cap U(\mathbf{g})$ contains $\mathfrak{p}$, set

$$
\begin{aligned}
& \left(p^{l}(\mathbf{f}) \mid A^{l}(\mathbb{S}, \tau)\right)_{l^{l}(\mathfrak{p})}=\xi_{i}\left(\partial / \partial u_{i}\right)+\xi_{\lambda}\left(\partial / \partial v_{\lambda}^{l}\right), \\
& \left(p^{l}(\mathbf{g}) \mid A^{l}(\hat{G}, \tau)\right)_{l^{l}(\mathfrak{p})}=\eta_{i}\left(\partial / \partial u_{i}\right)+\eta_{\lambda}\left(\partial / \partial v_{\lambda}^{l}\right) .
\end{aligned}
$$

Since $\omega_{i}$ is invariant under $p^{l}(\mathbb{S}, \tau),\left\langle\omega_{i}, p^{l}(\mathbf{f})\right\rangle$ is a constant for $\mathbf{f}$ in $\mathfrak{L}$. Hence the standard relation between the exterior derivative of Pfaffian forms and the bracket of vector fields imply immediately the following: If we set

$$
\left(p^{l}([\mathbf{f}, \mathbf{g}]) \mid A^{l}(\&, \tau)\right)_{l^{l}(\mathfrak{p})}=\zeta_{i}\left(\partial / \partial u_{i}\right)+\zeta_{\lambda}\left(\partial / \partial v_{\lambda}^{l}\right),
$$

then

$$
\zeta_{i}=c_{i}^{j k} \xi_{j} \eta_{k}+a_{i}^{j \lambda}\left(\xi_{j} \eta_{\lambda}-\xi_{\lambda} \eta_{j}\right) .
$$

Lemma IV.3. Let $\mathbf{f}$ and $\mathbf{g}$ be vector field elements in a domain $U$ in $\mathbf{R}^{n}$. Assume that $U(\mathbf{f}) \cap U(\mathbf{g})$ contains the origin, 0 , of $\mathbf{R}^{n}$. Set $\mathbf{f}_{j}^{j_{1} \cdots j_{\nu}}=\partial^{\nu} \mathbf{f}_{i} / \partial x_{j_{1}}$ $\cdots \partial x_{j_{v}}$ and $\mathbf{g}_{j}^{j_{1} \cdots j_{v}}=\partial^{\nu} \mathbf{g}_{j} / \partial x_{j_{1}} \cdots \partial x_{j_{\nu}}$, and $p^{l}([\mathbf{f}, \mathbf{g}])=\mathbf{h}_{j}\left(\partial / \partial x_{j}\right)+\mathbf{h}_{j}^{j_{1} \cdots j_{\nu}}$ $\left(\partial / \partial p_{j}^{j_{1} \cdots j_{\nu}}\right)$, where $(x, y, p)$ is the coordinate system in $G^{l}(U)$ associated with $(x)$ in $U$. Then

$$
\begin{aligned}
& \left(p^{l}(\mathbf{f})\right)_{I^{l_{0}}(0)}=\mathbf{f}_{j}(0)\left(\partial / \partial x_{j}\right)-\mathbf{f}_{j}^{j_{1} \cdots j_{\nu}}(0)\left(\partial / \partial p_{j}^{j_{1} \cdots j_{\nu}}\right),
\end{aligned}
$$

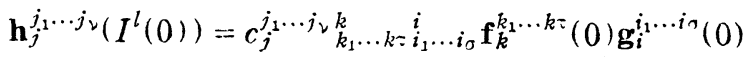

$$
\begin{aligned}
& \left.-\mathbf{f}_{j}^{j_{1} \ldots j_{\nu} k}(0) \mathbf{g}_{k}(0)+\mathbf{g}_{j}^{j_{1} \ldots j_{\nu k}}, 0\right) \mathbf{f}_{k}(0),
\end{aligned}
$$

where c's are given in Lemma IV. 2.

Proof. Take a one-parameter family $f^{t}$ of homeomorphism elements in $U$, with $f^{\circ}=$ the identity mapping, such that $\mathbf{f}$ is equal to $\left(d f^{t} / d t\right)_{t=0}$ on a neig- 
hborhood of 0 . Then $p^{l}(\mathbf{f})$ is equal to $\left(d p^{l}\left(f^{t}\right) / d t\right)_{t=0}$ on a neighborhood of $I^{l}(0)$. Hence $\left(p^{l}(\mathbf{f})\right)_{I^{l}(0)}$ is equal to the tangent of the curve $p^{l}\left(f^{t}\right)\left(I^{l}(0)\right)$ $=j_{0}^{l}\left(f^{t}\right)^{-1}$ at $t=0$. Then the first part follows immediately. The second part is equal to the formula (9) in the case $B$ consists of all homeomorphism elements of $U$.

Lemma IV. 4. Let $(\mathfrak{B}, \mathfrak{p})$ be a local continuous pseudo-group of M. Assume that $\mathbb{B}$ is transitive. Let $G$ be a parameter infinite Lie $(F)$-group of $(\mathbb{G}, \mathfrak{b})$. Denote by $L$ the infinite Lie $(F)$-algebra associated with $G$. Then there is an admissible filtration $C^{(l)}$, ( $c f$. Def. I.4), of the parameter space $H$ of $L$ with the following properties:

$\left(1^{\circ}\right)$ For any $\xi$ in $C^{(l)}$, and for anv $\eta$ in $H,[\xi, \eta]$ is in $C^{(l-1)}$ for each integer $l$.

$\left(2^{\circ}\right)$ The dimension of $H / C^{(l)}$ is $n_{l}$, where $n_{l}$ is the dimension of $A^{l}(\mathbb{B}, \mathfrak{b})$ $=\left\{X \in A^{l}(\$) ; \beta(X)=\mathfrak{p}\right\}$.

$\left(3^{\circ}\right)$ We can find a sequence $\xi^{1}, \xi^{2}, \ldots$ of elements in $H$ such that

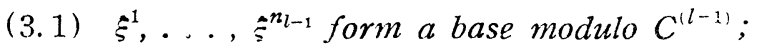

(3.2) we can find $\eta_{l}^{1}, \ldots, \eta_{l}^{m_{l}}$ in $C^{(l-1)}$ so that $\xi^{1}, \ldots, \xi^{n_{l-1}}, \eta_{l}^{1}, \ldots, \eta_{l}^{m_{l}}$ form a base of $\dot{H}$ modulo $C^{(l)}$ and

$$
\begin{aligned}
{\left[\xi^{j}, \xi^{k}\right] } & \equiv c_{i}^{j k} \xi^{i} & & \left(\bmod C^{(l-1)}\right), \\
{\left[\xi^{j}, \eta_{l}^{\lambda}\right] } & \equiv a_{i}^{j \lambda} \xi^{i} & & \left(\bmod C^{(l-1)}\right), \\
{\left[\eta_{l}^{\lambda}, \eta_{l}^{\lambda^{\prime}}\right] } & \equiv 0 & & \left(\bmod C^{(l-1)}\right),
\end{aligned}
$$

where $c_{i}^{j k}$ and $a_{i}^{j \lambda}$ are given in Theorem IV.5.

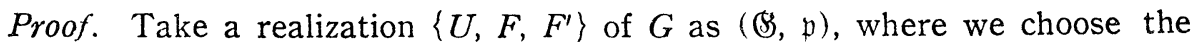
same coordinate system as in Theorem IV. 5. Then $\left\{U,(d F)_{0},\left(d F^{\prime}\right)_{0}\right\}$ is a realization of $L$ as $(\Omega, p)$. Denote by $C^{(l)}$ the set of all $\xi$ in $H$ such that each component of $(d F)_{0}(\xi)$ is in $H_{n}^{n(l+1)}$ (cf. Example 2, 1 Chap 1). (Remark that, $(d F)_{0}$ being linear, is everywhere defined). Then by Prop. I. $3 C^{(l)}$ is an admissible filtration of $H$. By the definition of the parameter infinite Lie $(F)$-algebra, Lemma IV. 3 implies that $\xi$ is in $C^{\prime l)}$ if and only if $\left(p^{l}(\mathbf{f}) \mid A^{l}(\mathfrak{B}, \mathfrak{p})\right)_{I^{l}(\mathfrak{p})}=0$ for a vector field element $\mathbf{f}$ corresponding to $\xi$, where $\mathbf{f}=\mathbf{f}_{j}\left(\partial / \partial x_{j}\right)$ and $\mathbf{f}_{j}$ is the $j$-th component of $(d F)_{0}(\xi)$. Since $(B)$ is transitive, for any tangent vector $Z$ at $I^{l}(p)$ to $A^{l}((s, p)$ there is a vector field element $\mathbf{f}$ in $\mathfrak{Q}$ such that $Z=\left(p^{l}(\mathbf{f})\right)_{1^{l}(p)}$. This proves (2). ( $\left.1^{\circ}\right)$ follows from (7) and (9). Take $\xi^{i}$ and 
$\eta_{l}^{\lambda}$ in $H$ such that $\xi^{i}$ and $\eta_{l}^{\lambda}$ correspond to vector field elements $f^{i}$ and $\mathrm{g}_{l}^{\lambda}$ with the condition $\left(p^{l}\left(\mathbf{f}^{i}\right) \mid A^{l}((\mathfrak{S}, \mathfrak{p}))_{l} l_{(\mathfrak{p})}=\left(\partial / \partial u_{i}\right)\right.$ and $\left(p^{l}\left(\mathbf{g}_{l}^{\lambda}\right) \mid A^{l}(\mathbb{S}, p)\right)_{1} l_{(\mathfrak{p})}=\left(\partial / \partial v_{l}^{\lambda}\right)$, respectively, where $u_{i}$ and $v_{l}^{\lambda}$ are coordinate functions employed in the formula (7). Then $\left(3^{\circ}\right)$ follows from (7) and (9).

\section{§3. Prolongations}

Let $\left(M_{1}, M, \rho\right)$ be a fibered manifold. Let $\mathcal{G}_{1}$ and $\mathbb{S}$ be continuous pseudogroups in $M_{1}$ and $M$, respectively. These notations will be kept throughout this section. Take homeomorphism elements $g$ and $f$ of $M_{1}$ and $M$, respectively. We say that $g$ is a prolongation of $f$ with respect to the fibered manifold ( $M_{1}$, $M$, $\rho)$ when $\rho U(g)=U(f)$ and $\rho^{\circ} g=f \circ \rho$. Take an open set $U$ in $M$. Let $f^{y}$ be a family on $U$ in $\left(\mathbb{S}\right.$, with a parameter manifold $M^{\prime}$. A family $g^{. \prime}$ on an open set $V$ in $\Theta_{1}$, with the parameter manifold $M^{\prime}$, is called a covering family of $f^{y}$ with respect to $\left(M_{1}, M, \rho\right)$, when $\rho(V)=U$ and $g^{y}$ is a prolongation of $f^{y}$ for any point $y$ in $M^{\prime}$.

Definition IV.7. $\mathscr{B}_{1}$ is called an isomorphic prolongation of $\mathbb{B}$ with respect to $\left(M_{1}, M, \rho\right)$, if for any element $g$ of $\mathbb{S}_{1}$ and for any $x$ in $U(g)$ there is a neighborhood $V$ of $x$ such that $g \mid V$ is a prolongation of an element of $B$ and if there is an integer $l_{1}$ satisfying the following conditions:

$\left(1^{\circ}\right)$ For any point $x$ in $M_{1}$, we can choose a neighborhood $\mathscr{U}$ of $I^{l_{1}}(\rho(x))$ in $G^{l_{1}}(M)$ and a neighborhood $U_{1}$ of $x$ in $M_{1}$ in such $a$ way that there is $a$ covering family $g^{t}$ of $f^{t}$ on $U_{1} \cap \rho^{-1}(U)$ in $\mathbb{S}_{1}$ for any one-parameter family $f^{t}$ on an open set $U$ in $\mathbb{S}$ such that $j^{l_{1}}\left(f^{t}\right) \subset \mathcal{U}$.

$\left(2^{\circ}\right)$ If $f$ is in $\mathbb{S}^{-}, j_{y}^{l_{1}}(f)=I^{l_{1}}(y)$ for a point $y$ in $U(f)$, and if $g$ is a prolongation of $f$ in $\mathbb{B}_{1}^{-}$, then $g(x)=x$ for any $x$ in $U(g)$ such that $\rho(x)=y$.

In the remainder of this section, $\mathscr{S}_{1}$ is always assumed to be an isomorphic prolongation of $\mathbb{B}$. It is clear that $p^{l}(\mathbb{B}, \tau)$ is an isomorphic prolongation of the restriction of $\mathbb{S}^{\prime}$ to $\tau^{-1}\left(U^{\prime}\right)$, where $U^{\prime}$ is an open set in $M^{\prime}$ and $\tau$ is a crosssection of $\left(M, M^{\prime}, \pi\right)$ over $U^{\prime},\left(M, M^{\prime}, \pi\right)$ being the fibered manifold of invariants of $\mathbb{B}$.

Proposition IV.9. Let $f$ be an element of $\$$. Let $g_{1}$ and $g_{2}$ be elements in $\mathbb{B}_{1}$. If both $g_{1}$ and $g_{2}$ are prolongations of $f$, then there is an element in $\mathfrak{O}_{1}^{-}$ such that $g$ is an extension of both $g_{1}$ and $g_{2}$. 
Proof. It is sufficient to show that $g_{1}$ coincides with $g_{2}$ on $U\left(g_{1}\right) \cap U\left(g_{2}\right)$. Assume that it is not empty. Then $g_{1} \circ g_{2}^{-1}$ is a prolongation of an identity mapping $f \circ f^{-1}$. Therefore, by $\left(2^{\circ}\right)$ in Def. IV.7 it follows that $g_{1}$ and $g_{2}$ coincides on $U\left(g_{1}\right) \cap U\left(g_{2}\right)$.

Let $U$ be an open submanifold of $M$. Denote by $(S \mid U$ the collection of all homeomorphism elements in $\mathbb{S}^{-}$such that their domains and images are in $U$. Clearly, $\mathbb{B} \mid U$ is a continuous pseudo-group in $U$. Let $\left(N, M, \rho^{\prime}\right)$ be a fibered manifold. Denote by $N \oplus A^{l}(\$)$ the submanifold of $N \times A^{l}(\$)$ consisting of all points $(v, X)$ such that $\rho^{\prime}(v)=\beta(X)$. Denote by $\alpha^{\prime}$ the mapping of $N \oplus A^{l}(\dot{\xi})$ onto $M$ defined by the formula: $\alpha^{\prime}(v, X)=\alpha(X)$. $\left(N \oplus A^{l}(\$), M, \alpha^{\prime}\right)$ is a fibered manifold. For any $f$ in $\left(B\right.$, denote by $p_{v}^{l}(f)$ the homeomorphism element of $N \oplus A^{l}(\$)$, of domain $\left(\alpha^{\prime}\right)^{-1}(U(f))$, defined by the formula: $\left[p_{N}^{l}(f)\right](v, X)$ $=\left(v, p^{l}(f)(X)\right)$. By Prop. IV. 6 and 7, Theorem IV.2, it is clear that the collection of all $p_{N}^{l}(f)$, when $f$ runs through in $B$, furms a continuous pseudogroup in $N \oplus A^{l}(\mathbb{B})$, which we shall denote by ${ }^{\prime} p^{l}\left(N\right.$; $(B)$. Denote by $B^{l}(N ;$ (B) the submanifold of $N \oplus A^{l}(\dot{S})$ consisting of all points $\left(v, I^{l}\left(\rho^{\prime}(v)\right)\right)$ with $v$ in $N$.

THEOREM IV.6. Let $\left(B_{1}\right.$ be an isomorphic prolongation of $B$ with respect to $\left(M_{1}, M, \rho\right)$. Then, for any sufficiently large integer $l$, there is an open connected neighborhood $\mathscr{Y}^{*}$ of $B^{l}\left(M_{1}\right.$; $\left.\$\right)$ in $M_{1} \oplus A^{l}(\$)$ and there is a mapping $h$ of $\mathscr{U}^{*}$ onto $M_{1}$ with the following conditions: $\left(1^{\circ}\right)\left(\mathscr{U}^{*}, M_{1}, h\right)$ is a fibered manifold; $\left(2^{\circ}\right){ }^{l} p^{l}\left(M_{1} ;(\mathfrak{B}) \mid \mathscr{U}^{*}\right.$ is an isomorphic prolongation of $\hat{G}_{1}^{-}$, with respect. to $\left(\mathscr{U}^{*}, M_{1}, h\right)$.

Proof. Take $v_{0}$ in $M_{1}$. Take open neighborhoods $\mathscr{U}$ of $I^{l}\left(\rho\left(v_{0}\right)\right)$ in $G^{\prime}(M)$, $U_{1}$ of $v_{0}$ in $M_{1}$ satisfying the condition $\left(1^{\circ}\right)$ in Def. IV.7. Set $\mathscr{U}^{k} v_{0}^{k}=\left(M_{1}\right.$ $\oplus A^{l}((\$)) \cap\left(U_{1} \times \mathscr{Y}^{\prime}\right)$. Clearly, ${ }^{\prime} \mathscr{L}_{v_{0}}^{*}$ is a neighborhood of $\left(v_{0}, I^{\prime}\left(\rho\left(v_{0}\right)\right)\right.$. Take $(v, X)$ in ${ }^{\prime} \mathscr{\ell}_{v^{\prime}}^{*}$, and choose $f$ in $\mathbb{S}$ such that $X=j_{p^{\prime}}^{l}(f)^{-1}$. By $\left(1^{\cup}\right)$ in Def. IV.7, there is $g$ in $G_{1}$ which is a prolongation of $f$ and such that $U(g) \equiv v$. Set $h(v, X)=g(v)$. By the same argument as in the proof of Prop. IV.9 we see that $h(v, X)$ is independent of the choice of $f$ and $g$. Moreover the second part of $\left(1^{\circ}\right)$ in Def. IV. 7 shows that $h$ is a real analytic mapping of $\mathscr{U}_{v_{0}}^{*}$ into $M_{\mathrm{i}}$. Moreover $h\left(v, I^{l}(\rho(v))=v\right.$. Therefore, if we choose a suitable open neighborhood $\mathscr{U}_{v_{0}}^{*}$ of $\left(v_{0}, I^{l}\left(\rho\left(v_{0}\right)\right)\right.$ in $\mathscr{U}_{v_{0}}^{*},\left(\mathscr{U}_{v_{0}}^{*}, U_{1}, h\right)$ is a fibered manifold. Let $g$ be an element of $\mathscr{G}_{1} . \quad g$ is a prolongation of an element $f \in \mathbb{S}$. Then 
the definition of $h$ implies that $h\left(\left[p_{N}^{l}(f)\right](v, X)\right)=g(h(v, X))$. Then it is easy to check that ${ }^{l} p^{l}\left(M_{1} ; \mathfrak{B}\right) \mid \mathscr{U}_{v_{0}}^{*}$ is an isomorphic prolongation of $\mathscr{G}_{1} \mid U_{1}$. The above definition of $h$ is intrinsic, and so they must coincide on $\mathscr{U}_{v}^{*} \cap \mathscr{U}_{v^{\prime}}^{*}$. Therefore choosing a suitable open neigborhood of $B^{l}\left(M_{1}\right.$; (B), we obtain $\mathscr{C l}^{*}$ and $h$ as in the theorem. q.e.d.

Take $f$ in $\&$ such that $j^{l}(f)$ is sufficiently near $\left\{I^{l}(w) ; w \in U(f)\right\}$. If $g$ is a prolongation of $f$ in $\mathbb{S}_{1}$, we see by the definition

$$
g(v)=h\left(v, j_{p(v)}^{l}(f)^{-1}\right) .
$$

Therefore we have the following two propositions.

Proposition IV.10. For any integer $l_{1}$ there is an integer $l_{2}$ with the follow. ing conditions: (1) For any neighborhood $\mathscr{U}_{1}$ of $I^{l_{1}}(v)$ in $G^{l_{1}}\left(M_{1}\right)$ there is a neighborhood $\mathscr{U}$ of $I^{l_{2}}(\mu(v))$ in $G^{l_{2}}(M)$ such that $j^{l_{1}}(g) \cap \alpha^{-1} \circ \alpha\left(\mathscr{U}_{1}\right) \subseteq \mathscr{U}_{1}$, for any prolongation $g$ of $f$ where $f$ is an arbitary element in $\mathbb{S}^{-}$with $j^{l_{2}}(f) \subseteq \mathscr{U}$; (2) If $f$ is in $\mathbb{S}^{-}, j_{w}^{I_{2}}(f)=I^{I_{2}}(w)$ for a point $w$ in $U(f)$, and if $g$ is a prolongation of $f$ in $\mathbb{G}_{1}$, then $j_{v}^{l_{1}}(g)=I^{l_{1}}(v)$ for any $v$ in $U(g)$ such that $\rho(v)=w$.

Proposition VI.11. Condition $\left(1^{\circ}\right)$ in Def. IV.7 holds for any family in (S), not necessarily only for one-parameter family in $\mathbb{B}$.

By Prop. IV.10, we see easily the following:

Proposition IV.12. Let $\left(M_{2}, M_{1}, \rho_{1}\right)$ be a fibered manifold. Assume that a continuous pseudo-group $\mathbb{Q}_{2}$ of $M_{2}$ is an isomrophic prolongation of $\mathbb{G}_{1}$ with respect to $\left(M_{2}, M_{1}, \rho_{1}\right)$. Then $\mathbb{S}_{2}$ is an isomorphic prolongation of $\mathbb{B}$ with respert to $\left(M_{2}, M, \rho^{\circ} \rho_{1}\right)$.

\section{§4. Infinite continuous pseudo-gropus}

Let $\left(\mathscr{G}_{1}, \mathfrak{p}_{1}\right)$ and $\left(\mathfrak{G}_{2}, \mathfrak{p}_{2}\right)$ be local continuous pseudo groups in $M_{1}$ and $M_{2}$, respectively.

Definition IV.8. $\left(\mathfrak{G}_{2}, \mathfrak{p}_{2}\right)$ is said to be an isomorphic prolongation of $\left(\mathbb{S}_{1}\right.$, $H_{1}$ ) if there are open neighborhoods $U_{1}$ and $U_{2}$ of $p_{1}$ and $p_{2}$ in $M_{1}$ and $M_{2}$, respectively, and if there is a mapping $\rho_{1}$ of $U_{2}$ onto $U_{1}$ with the following conditions: (1) $\left(U_{2}, U_{1}, \rho_{1}\right)$ is a fibered manifold $(2) \mathbb{S}_{2} \mid U_{2}$ is an isomorphic prolongation of $\left(\dot{S}_{1} \mid U_{1}\right.$ with respect to $\left(U_{2}, U_{1}, \rho_{1}\right)$.

Proposition IV.13. If $\left(\mathbb{S}_{3}, \mathfrak{p}_{3}\right)$ is an isomorphic prolongation of $\left(\mathbb{S}_{2}, \mathfrak{p}_{2}\right)$. 
and if $\left(\mathbb{S}_{2}, p_{2}\right)$ is an isomorphic prolongation of $\left(\left(\mathbb{S}_{1}, \mathfrak{p}_{1}\right)\right.$, then $\left(\mathbb{G}_{3}, \mathfrak{p}_{3}\right)$ is an isomorphis prolongation of $\left(\mathbb{S}_{1}, \mathfrak{p}_{1}\right)$.

Proof. This is an easy corollary of Prop. IV. 12.

Proposition IV.14. If both $\left(\mathbb{G}_{1}, \mathfrak{p}_{1}\right)$ and $\left(\mathbb{B}_{2}, \mathfrak{p}_{2}\right)$ are isomorphic prolongations of $(\mathfrak{S}, \mathfrak{p})$, there is a local continuous pseudo-group $\left(\mathbb{S}^{*}, \mathfrak{p}^{*}\right)$ which is an isomorphic prolongation of both $\left(\mathbb{S}_{1}, \mathfrak{p}_{1}\right)$ and $\left(\mathbb{S}_{2}, \mathfrak{p}_{2}\right)$.

Proof. It is easy to construct such $\left(\mathbb{S}^{*}, \mathfrak{p}^{*}\right)$ because of theorem IV. 6.

Definition IV.9. $\left(\mathbb{S}_{1}, \mathfrak{p}_{1}\right)$ is said to be equivalent to $\left(\mathbb{S}_{2}, \mathfrak{p}_{2}\right)$ if there is a local continuous pseudo-group $(\mathbb{S}, \mathfrak{p})$ such that $(\mathbb{S}, \mathfrak{p})$ is an isomorphis prolongation of both $\left(\mathbb{S}_{1}, \mathfrak{p}_{1}\right)$ and $\left(\mathbb{S}_{2}, \mathfrak{p}_{2}\right)$.

By Prop. IV. 13 and 14, we see easily that this definition of an equivalence actually gives an equivalence relation.

Definition IV.10. An equivalence class of the equivalence relation in Def. IV. 9 is called an infinite continuous pseudo-group. If $(\mathfrak{G}, \mathfrak{b )}$ is in an equivalence class $\mathscr{G},(\mathfrak{G}, \mathfrak{p})$ is said to be a representative of $\mathscr{G}$.

Let $B$ be a transitive continuous pseudo-group in a manifold $M$. Let $\mathfrak{p}_{1}$ and $\mathfrak{p}_{2}$ be points in $M$. Then $\left(\mathbb{S}, \mathfrak{p}_{1}\right)$ and $\left(\mathfrak{B}, \mathfrak{p}_{2}\right)$ are equivalent. Therefore the infinite continuous pseudo-group which has a representative $\left(\mathbb{B}, p_{1}\right)$ is said to be the infinite continuous pseudo-group determined by $(\$)$.

Definition IV.11. A continuous pseudo-group $\mathbb{B}$ of a domain $D=\left\{\left(x_{1}, \ldots\right.\right.$, $\left.\left.x_{N}\right)\right\}$ is said to be a Cartan's continuous pseudo-group of $D$, if there is an integer $h \geq 0$ and if there is a base $\omega_{1}, \ldots, \omega_{n}, \pi_{1}, \ldots, \pi_{m}(N=n+m)$ of Pfaffian forms on $D$, with the following conditions:

$\left(1^{\circ}\right) \quad \omega_{1}=d x_{1}, \ldots, \quad \omega_{h}=d x_{h}$,
$\left(2^{\circ}\right) \quad d \omega_{i}=\frac{1}{2} c_{i}^{j k} \omega_{j} \wedge \omega_{k}+a_{\imath}^{j \lambda} \omega_{j} \wedge \pi_{\lambda}, \quad c_{i}^{j k}+c_{i}^{k j}=0$, $(i, j, k=1, \ldots, n ; \lambda=1, \ldots, m)$,

where $c_{i}^{j k}$ and $a_{i}^{i \lambda}$ are functions of $x_{1}, \ldots, x_{h}$;

$\left(3^{\circ}\right)$ Let $\rho_{1}$ and $\rho_{2}$ be the projection of $D \times D$ onto the first and second facters, respectively. Then the exterior defferential system generated by 


$$
\begin{array}{cc}
x_{r} \circ \rho_{2}-x_{r} \circ \rho_{1} & (r=1, \ldots, h), \\
\rho_{2}^{*} \omega_{i}-\rho_{1}^{*} \omega_{i} & (i=1, \ldots, n)
\end{array}
$$

on $\left(D \times D, D, \rho_{1}\right)$ is in involution at any point in the diagonal;

$\left(4^{\circ}\right)$ A homeomorphism element $f$ of $D$ is in $B$ if and only if $x_{1}, \ldots, x_{h}$ and $\omega_{1}, \ldots, \omega_{n}$ are invariant under $f$.

The system consists of functions $x_{1}, \ldots, x_{h}$ and of Pfaffian forms $\omega_{1}, \ldots$, $\omega_{n}, \pi_{1}, \ldots, \pi_{m}$ will be called a Cartan's system. The equations in $\left(2^{\circ}\right)$ will be called the structure equations of $(\$$. Let us agree that, when we say that $(B$ is a Cartan's continuous pseudo-group in $D$, a choice of Cartan's system which defines $B$ is made and associated with $\mathbb{B}$.

Theorem IV.7. Let $\mathscr{G}$ be an infinite continuous pseudo-group. Then $\mathscr{G}$ has a representative $(\mathbb{S}, \mathfrak{p})$ such that $\mathbb{B}$ is a Cartan's continuous pseudo-group.

Proof. Let $\left(\mathbb{S}^{\prime}, \mathfrak{p}^{\prime}\right)$ be a representative of $\mathscr{G}$. Let $\tau$ be a cross-section of the fibered manifold $\left(M^{\prime}, M^{\prime \prime}, \pi\right)$ of invariants of $\mathbb{S}^{\prime}$ over on open neighborhood $U^{\prime}$ of $\pi\left(p^{\prime}\right)$. Since $p^{l}\left(\mathbb{S}^{\prime}, \tau\right)$ is an isomorphic prolongation of $\mathbb{S}^{\prime} \mid U$, where $U=\pi^{-1}\left(U^{\prime}\right)$, the theorem is an immediate colloary of Theorem IV.5.

\section{§5. Infinite continuous pseudo-groups and infinite Lie $(F)$-groups}

Let $G_{1}$ and $G_{2}$ be infinite Lie ( $F$ )-groups (cf. sentences preceeding Lef. IV. 5 ). Denote by $H_{1}$ and $H_{2}$ the parameter spaces of $G_{1}$ and $G_{2}$, respectively. We say that $G_{1}$ and $G_{2}$ are convergently isomorphic if there are convergent formal analytic mappings $F$ and $F^{\prime}$ of $H_{1}$ into $H_{2}$ and of $H_{2}$ into $H_{1}$, respectively, such that $F$ and $F^{\prime}$ establish as isomorphism of $G_{1}$ and $G_{2}$.

Proposition IV.15. Let $\left(\xi_{r}, p_{r}\right)$ be a local continuous pseudo-group of a manifold $M_{r},(r=1,2)$. Denote by $G_{r}$ a parameter infinite Lie (F)-group of $\left(\mathfrak{G}_{r}, \mathfrak{p}_{r}\right)$. If $\left(\mathfrak{G}_{1}, \mathfrak{p}_{1}\right)$ and $\left(\mathfrak{G}_{2}, \mathfrak{p}_{2}\right)$ are equivatent, then $G_{1}$ and $G_{2}$ are convergently isomorphic.

Proof. It is sufficient to prove the case when $\mathfrak{G}_{2}$ is an isomorphic prolongation of $\mathscr{G}_{1}$. We can assume then, without loss of generality, that-(1) $M_{1}$ is a domain $\left\{\left(x_{1}, \ldots, x_{n}\right) ;\left|x_{j}\right|<\varepsilon\right\}$, (2) $M_{2}=M_{1} \times W$, where $W$ is a domain in $m$-dimesional space. (3) $\mathfrak{p}_{1}$ and $\mathfrak{p}_{2}$ are origins of $M_{1}$ and $M_{2}$, respectively, and that $(4) \mathfrak{G}_{2}$ is an isomorphic prolongation of $\mathscr{G}_{1}$ with respect to $\left(M_{1} \times W\right.$, 
$\left.M_{1}, \rho\right)$ where $\rho$ is the canonical prolojection. We can assume also that there are realization (cf. Def. IV.6) $\left(M_{r}, F_{r}, F_{r}^{\prime}\right)$ of $G_{r}$ as $\left(G_{r}, p_{r}\right)$. Let $F^{\prime \prime}$ be the convergent formal analytic mapping of $H_{n+m}^{n+m}$ into $H_{n}^{n}$ defined by the formula: $F^{\prime \prime}(\xi)=\left(\xi_{1}\left(x_{1}, \ldots, x_{n}, 0, \ldots, 0\right), \ldots, \xi_{n}\left(x_{1}, \ldots, x_{n}, \ldots, 0\right)\right) \in H_{n}^{n},\left(\xi=\left(\xi_{1}\right.\right.$, $\left.\left.\ldots, \xi_{n+m}\right) \in H_{n+m}^{n+m}\right)$. Then it is clear by the definitions that there is a unique convergent formal analytic mapping $F$ of $H_{2}$ into $H_{1}$ such that $F_{1} \circ F=F^{\prime \prime} \circ F_{2}$ and such that $F$ is a homomorphism of $G_{2}$ into $G_{1}$. The existence of the convergent inverse of $F$ follows from the existence of the mapping $h$ as in Theorem IV.6 (cf. (10)).

Definition IV.12. Let $\mathbb{S}_{r}$ be a Cartan's continuous transitive pseudo-group in a domain $D_{r},(r=1,2)$. Denote by $\varphi_{1}, \ldots, \varphi_{n^{\prime}}, \psi_{1}, \ldots, \psi_{m}$ and by $\omega_{1}$, $\ldots, \omega_{n}, \pi_{1}, \ldots, \pi_{m}$ Cartan's systems associated with $\mathbb{B}_{1}$ and $\mathbb{B}_{2}$, respectively. Let

$$
d \omega_{i}=\frac{1}{2} c_{i}^{j k} \omega_{j} \wedge \omega_{k}+a_{i}^{j \lambda} \omega_{j} \wedge \pi \lambda
$$

be the structure equations of $\mathrm{G}_{2}$, where $c_{i}^{j k}$ and $a_{i}^{j \lambda}$ are constants. We say that $\mathbb{S}_{2}$ is subordinate to $\mathbb{S}_{1}$ if there are linear combinations

$$
\begin{aligned}
& \Omega_{i}=t_{i}^{q} \varphi_{q}, \\
& \Pi_{\lambda}=t_{\lambda}^{q} \varphi_{q}+u_{\lambda}^{o} \psi_{\sigma}
\end{aligned}
$$

where t's and $u$ 's are constants, with the following conditons:

(1) $\Omega_{1}, \ldots, \Omega_{n}, \Pi_{1}, \ldots, I_{m}$ are linearly independent at each point of $D_{1}$;

(2) $d \Omega_{i}=\frac{1}{2} c_{i}^{j k} \Omega_{j} \wedge \Omega_{k}+a_{i}^{j \lambda} \Omega_{j} \wedge \Pi_{\lambda}$

A choice of the linear combinations, $\Omega_{i}$ and $\Pi_{\lambda}$, which satisfy (1) and (2) will be called a subordination of $\mathbb{S}_{2}$ to $\mathbb{B}_{1}$. Let $\rho$ be a mapping of an open set $U_{1}$ of $D_{1}$ onto an opeu set $U_{2}$ of $D_{2} . \quad \rho$ is called an integral of the subordination if it satisfy the following conditions:

$\left(1^{\circ}\right) \quad \rho^{*} \omega_{i}=\Omega_{i} \quad(i=1, \ldots, n)$,

$\left(2^{\circ}\right)\left(U_{1}, U_{2}, \rho\right)$ is a fibered manifold.

Theorem IV. 8. Let $\left(\leftrightarrow_{r}, \mathfrak{p}_{r}\right)$ be a local Cartan's continuous transitive pseudogroup of a domain $D_{r}(r=1,2)$. Assume that $\mathbb{B}_{2}$ is subordinate to $\mathbb{B}_{1}$. Then for any subordination of $\mathbb{G}_{2}$ to $\mathbb{B}_{1}$ we have the following: 
$\left(1^{\circ}\right)$ There is its integral $\rho$ such that $\rho$ is defined at $p_{1}$ and such that $\rho\left(p_{1}\right)=p_{2}$.

$\left(2^{\circ}\right)$ If $\rho^{\prime}$ is another such integral, there is an element $f$ in $\left(\left(_{\aleph_{2}}\right)_{\mathfrak{p}_{2}}\right.$ such that $\rho^{\prime}$ is equal to $f \circ \rho$ on a neighborhood of $\mathfrak{p}_{1}$.

$\left(3^{\circ}\right)$ Any homeomorphism element of $\mathbb{B}_{1} \mid U_{1}$ is a prolongation of an element in $\mathbb{B}_{2} \mid U_{2}$ with respect to $\left(U_{1}, U_{2}, \rho\right)$.

Proof. Let $\rho_{1}^{\prime}$ and $\rho_{2}^{\prime}$ be the projection of $D_{1} \times D_{2}$ onto the first and the second factors, respectively. On $\left(D_{1} \times D_{2}, D_{1}, \rho_{1}^{\prime}\right)$, consider the differential system $\Sigma^{\prime}$ generated by

$$
\rho_{2}^{\prime *} \omega_{i}-\rho_{1}^{\prime *} \Omega_{i}
$$

We denote by $\Sigma$ the differential system on $\left(D_{2} \times D_{2}, D_{2}, \rho_{1}\right)$ defined in $\left(3^{\circ}\right)$ in Def. IV.11 for $\mathbb{G}_{2}$. Then a mapping $\rho$ of an open neighborhood $U_{1}$ of $p_{1}$ onto an open neighborhood $U_{2}$ of $k_{2}$ is an integral of the subordination if and only if $\left(U_{1}, U_{2}, \rho\right)$ is a fibered manifold and its graph is an integral of $\Sigma^{\prime}$.

Take a submanifold $D_{2}^{\prime}$ of $D_{1}$, passing through $p_{1}$, such that the restrictions of $\Omega_{i}, \Pi_{\lambda}$ to $D_{2}^{\prime}$ are linearly independent. Comparing with the polar equations of $\Sigma$, we find the following: The system $\Sigma^{\prime}$ as well as the restriction $\Sigma^{\prime \prime}$ of $\Sigma^{\prime}$ to $\left(D_{2}^{\prime} \times D_{2}, D_{2}^{\prime}, \rho_{1}\right)$ are in involution at $\left(p_{1}, p_{2}\right)$, and any integral manifold of $\Sigma^{\prime \prime}$ passing through $\left(p_{1}, p_{2}\right)$ can be locally extended uniquely to an integral manifold of $\Sigma^{\prime}$. Take an integral $\rho$ of $\Sigma^{\prime}$ such that $\rho\left(\mathfrak{p}_{1}\right)=\mathfrak{p}_{2}$. Then the condition $\rho^{*} \omega_{1}=\Omega_{1}$ implies that the restriction of $\rho$ to $D_{2}^{\prime}$ induces a homeomorphism of a neighborhood $U_{1}^{\prime}$ of $p_{1}$ in $D_{2}^{\prime}$ onto a neighborhood $U_{2}$ of $p_{2}$. In particular, $\left(U_{1}, U_{2}, \rho\right)$ is a fibered manifold. Therefore $\rho$ is an integral of the subordination. Thus $\left(1^{\circ}\right)$ is proved.

Let $\rho^{\prime}$ be another integral of the subordination. Denote by $\bar{\rho}$ and $\bar{\rho}^{\prime}$ the restricions of $\rho$ and $\rho^{\prime}$ to a small neighborhood of $p_{1}$ in $D_{2}^{\prime}$. Then they have inverses and $\omega_{i}=\left(\bar{\rho}^{-1}\right)^{*} \Omega_{i}=\left(\bar{\rho}^{\prime-1}\right)^{*} \Omega_{i}$. Take a homeomorphism element $f$ of $D_{2}$ such that $\dot{f} \circ \bar{\rho}=\bar{\rho}^{\prime}$ on a neighborhood of $\mathfrak{p}_{1}$ in $D_{2}^{\prime}$. Then it follows easily that $f^{*} \omega_{i}=\omega_{i}$. Hence $f$ is in $\mathcal{G}_{2}$. Therefore $f \circ \rho$ is again an integral of the subordination and coincides with $\rho^{\prime}$ on $D_{2}^{\prime}$. Hence, by the remark made earlier, $f \circ \rho=\rho^{\prime}$ on a neighborhood of $p_{1}$. Thus $\left(2^{\circ}\right)$ is proved. Because of the expression of $\Omega_{i}, \rho^{\circ} \mathrm{g}$ is again an integral of the subordination for any element $g$ in $\left(\xi_{1}\right.$. Then $\left(3^{\circ}\right)$ follows from $\left(2^{\circ}\right)$. This finishes the proof of Theorem IV. 8 . 
Let $\mathbb{B}$ r be a Cartan's transitive continuous pseudo-group, $(r=1,2,3)$. Denote by $\varphi_{1}, \ldots, \varphi_{n^{\prime}}, \psi_{1}, \ldots, \psi_{m^{\prime}}$ and $\omega_{1}, \ldots, \omega_{n}, \pi_{1}, \ldots, \pi_{m}$ Caran's systems associated with $\mathscr{G}_{1}$ and $\mathscr{G}_{2}$, respectively. Assume that $\mathscr{G}_{2}$ is subordinate to $\mathbb{G}_{1}$ and that $\mathbb{G}_{3}$ is subordinate to $\mathscr{S}_{2}$. Assume also that $t_{i}^{q} \varphi_{q}$ and $t_{\lambda}^{q} \varphi_{q}+u_{\lambda}^{\sigma} \varphi_{\sigma},{ }^{\prime} t_{j}^{i} \omega_{i}$ and ' $t_{\tau}^{i} \omega_{i}+{ }^{\prime} u_{\tau}^{\lambda} \pi_{\lambda}$ form subordinations of $\mathbb{B}_{2}$ to $\mathbb{G}_{1}$, of $\mathbb{G}_{3}$ to $\mathbb{S}_{2}$, resepctively. Then it is easy to see that ${ }^{\prime} t_{j}^{i} t_{i}^{q} \varphi_{q}$ and $\left({ }^{\prime} t^{i} t_{i}^{q}+{ }^{\prime} u_{\tau}^{\lambda} t_{\lambda}^{q}\right) \varphi_{q}+{ }^{\prime} u_{:}^{\lambda} u_{\lambda}^{\beta} \psi_{0}$ form a subordination of $\mathbb{G}_{3}$ to $\mathbb{G}_{1}$. This subordination is called the composition of the subordinations. If $\rho$ and $\rho^{\prime}$ are integrals of the subordinations of $\mathscr{G}_{2}$ to $\mathscr{G}_{1}$ and of $\mathbb{S}_{3}$ to $\mathbb{S}_{2}$, then $\rho^{\prime} \circ \rho$ is an integral of their composition.

Lemma IV.5. Let $\left(\mathbb{S}_{r}, \mathfrak{p}_{r}\right)$ be a Cartan's local transitive continuous pseudogroup in $D_{r},(r=1,2,3)$. Assume that $\mathbb{G}_{2}$ is subordinate to $\mathbb{B}_{1}$, and that $\mathbb{G}_{3}$ is subordinate to $\mathbb{G}_{2}$. Take subordinations $s_{1}$ and $s_{2}$ of $\mathscr{G}_{2}$ to $\mathbb{G}_{1}$ and of $\mathbb{G}_{3}$ to $\mathbb{S}_{2}$, respectively. Take a mapping $\pi$ of $D_{1}$ to $D_{3}$ such that $\left(D_{1}, D_{3}, \pi\right)$ is a fibered manifold and such that $\pi\left(p_{1}\right)=F_{3}$. Assume that $\mathfrak{G}_{1}$ is an isomorphic prolongation of $\mathbb{B}_{3}$ with respect to $\left(D_{1}, D_{3}, \pi\right)$ and that $\pi$ is an integral of the composition of the subordinations $s_{1}$ and $s_{2}$. Then $\left(\mathfrak{G}_{2}, p_{2}\right)$ is an isomorphic. prolongation of $\left(\mathbb{S}_{3}, p_{5}\right)$ with respect to any integral of the subordination $s_{2}$, which maps $\mathfrak{p}_{2}$ to $\mathfrak{p}_{3}$.

Proof. Let $\rho_{1}$ and $\rho_{2}$ be integrals of $s_{1}$ and $s_{2}$ such that $\rho_{1}\left(p_{1}\right)=p_{2}$ and $\rho\left(\mathfrak{k}_{2}\right)=\mathfrak{p}_{3}$, respectively. Since $\rho_{2} \rho \rho_{1}$ is an integral of the composition of $s_{1}$ and $s_{2}$, by $\left(2^{\circ}\right)$ in Theorem IV.8, we can assume that $\pi=\rho_{2}^{\circ} \rho_{1}$ on a neighborhood of $\mathfrak{p}_{1}$. By $\left(3^{\circ}\right)$ of Theorem IV.8, any element of $\mathbb{G}_{2}$ is an prolongation of an element of $B_{3}$. It is easy to verify the conditions $\left(1^{\circ}\right)$ and $\left(2^{\circ}\right)$ in Def. IV.7 for $\mathbb{G}_{2}$ and $\mathbb{B}_{3}$ with respect to $\rho_{2}$, by lifting first to $\mathscr{G}_{1}$ and then going down to $\mathbb{B}_{2}$.

Proposition IV.16. Let $\left((\xi, p)\right.$ and $\left(\left(\dot{S}^{\prime}, p^{\prime}\right)\right.$ be local transitive continuous pseudo-groups of $D$ and $D^{\prime}$, respectively. Denote by $G$ and $G^{\prime}$ parameter infinite Lie $(F)$-groups of $(\mathfrak{G}, p)$ and $\left(\mathbb{B}^{\prime}, \mathfrak{p}^{\prime}\right)$, respectively. If $G$ and $G^{\prime}$ are (formally) isomorphic, then $(\mathfrak{S}, \mathfrak{p})$ and $\left(\mathfrak{G}^{\prime}, \mathfrak{p}^{\prime}\right)$ are equivalent.

Proof. Let $F$ be a homeomorphism of $G$ onto $G^{\prime}$. Denote by $L$ and $L^{\prime}$ the associated infinite Lie $(F)$-algebras of $G$ and of $G^{\prime}$, respectively. Take admissible filtrations $H^{(l)}$ and $H^{\prime(l)}$ of the parameter space $H$ and $H^{\prime}$ of $L$ and $L^{\prime}$, respectively, which satisfy the conditions in Lemma IV.4. We can choose a 
positive integer $k$ such that $F_{0}\left(H^{(l)}\right) \subseteq H^{(l-h)}$ and such that $F_{0}^{-1}\left(H^{\prime(l)}\right) \subseteq H^{(l-k)}$, where $F_{0}=(d F)_{0}$ is the homeomorphism of $L$ onto $L^{\prime}$ induced by $F$. Set for sufficiently large fixed $l$

$$
\begin{aligned}
& \left(\mathbb{B}_{1}, \mathfrak{p}_{1}\right)=\left(p^{l+2 k}\left(\mathfrak{B}^{\prime}, \mathfrak{p}^{\prime}\right), I^{l+2 k}\left(\mathfrak{p}^{\prime}\right)\right), \\
& \left(\mathfrak{G}_{2}, \mathfrak{p}_{2}\right)=\left(p^{l+k}(\mathbb{S}, \mathfrak{p}), I^{l+k}(\mathfrak{p})\right), \quad\left(\mathfrak{G}_{3}, \mathfrak{p}_{3}\right)=\left(p^{l}\left(\mathfrak{S}^{\prime}, \mathfrak{p}^{\prime}\right), I^{l}\left(\mathfrak{p}^{\prime}\right)\right),
\end{aligned}
$$

(cf. $\left.(7)^{\prime}\right)$. If we show that $\left(\mathbb{S}_{r}, p_{r}\right),(r=1,2,3)$, defined above satisfy the conditions stated in Lemma IV. 5, then it follows that $\left(p^{l+k}(\mathbb{S}, p), I^{l+k}(p)\right)$ is an isomorphic prolongation of $\left(p^{l}\left(\mathbb{S}^{\prime}, \mathfrak{p}^{\prime}\right), I^{l}\left(\mathfrak{p}^{\prime}\right)\right)$, that is $(\mathbb{G}, \mathfrak{p})$ is equivalent to $\left(\mathbb{S}^{\prime}, p^{\prime}\right)$. In order to show that they actually satisfy the required conditions, let $D^{\left(l^{\prime}\right)}$ and $D^{\prime\left(l^{\prime}\right)}$ be sufficiently small neighborhoods of $I^{l^{\prime}}(\mathfrak{p})$ and $I^{l^{\prime}}\left(\mathfrak{p}^{\prime}\right)$, respectively. We define $\pi$ as the canonical projection of $D^{\prime^{(l+2 k)}}$ onto $D^{\prime(l)}$. Let $\omega_{1}, \ldots, \omega_{n}, \pi_{1}, \ldots, \pi_{n}$, where $n=n_{l+k-1}$ and $m=m_{l+k}$, be the base of Pfaffian forms on $D^{(l+k)}$ which satisfies the conditions in Theorem IV. 5 for $p^{l+k}(\mathbb{S}, \mathfrak{p})$. Denote by $\varphi_{1}, \ldots, \varphi_{n}, \psi_{1}, \ldots, \psi_{m^{\prime}}$, where $n^{\prime}=n_{l+2 k-1}^{\prime}$ and $m^{\prime}=m_{l+2 k}^{\prime}$ the base of Pfaffian forms on $D^{\prime(l+2 k)}$ which satisfies the conditions for $p^{l+2 k}\left(\mathbb{S}^{\prime}, \mathfrak{p}^{\prime}\right)$. Let

$$
\begin{aligned}
& d \omega_{i}=\frac{1}{2} c_{i}^{i^{\prime} i^{\prime \prime}} \omega_{i} \wedge \omega_{i^{\prime \prime}}+a_{i}^{i^{\prime} \lambda} \omega_{i} \wedge \pi_{\lambda}, \\
& d \varphi_{j}=\frac{1}{2}{ }^{j} c_{j}^{j, j^{\prime \prime}} \varphi_{j^{\prime}} \wedge \varphi_{j^{\prime \prime}}+{ }^{\prime} a_{j}^{j^{\prime} \sigma} \varphi_{j}, \wedge \varphi_{\sigma}
\end{aligned}
$$

be the structure equations. Choose $\xi^{1}, \ldots, \xi^{n}, \eta^{1}, \ldots, \eta^{m}$ in $H$ which satisfy the conditions in Lemma IV. 4 for the choice $\omega_{i}, \pi_{\lambda}$. We have the similar elements $\zeta^{1}, \ldots, \zeta^{n^{\prime}}, \mu^{1}, \ldots, \mu^{m^{\prime}}$ in $H^{\prime}$ for $\varphi_{j}, \psi_{\sigma}$. Then by Lemma IV. 4 ,

$$
\begin{aligned}
& {\left[\xi^{i^{\prime}}, \xi^{i^{\prime \prime}}\right] \equiv c_{i}^{i^{\prime} i^{\prime \prime}} \xi^{i}, \quad\left[\xi^{i \prime}, \eta^{\lambda}\right] \equiv a_{i}^{i^{\prime} \lambda} \xi^{i},} \\
& {\left[\eta^{\lambda}, \eta^{\lambda^{\prime}}\right] \equiv 0 \quad\left(\bmod H^{(l+k-1)}\right)}
\end{aligned}
$$

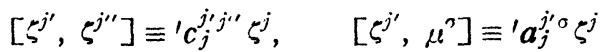

$$
\begin{aligned}
& {\left[\mu^{\sigma}, \mu^{\sigma^{\prime}}\right] \equiv 0 \quad\left(\bmod . H^{(l+2 k-1)}\right) \text {. }}
\end{aligned}
$$

Since $\mu^{\sigma}$ is in $H^{(l+2 k-1)}, F_{0}^{-1}\left(\ell^{\sigma}\right)$ is in $H^{(l+k-1)}$. Therefore we have

$$
\begin{aligned}
F_{0}^{-1}\left(\zeta^{j}\right) & \equiv t_{i}^{j} \xi^{i}+u_{\lambda}^{j} \eta^{\lambda}, \\
F_{0}^{-1}\left(\mu^{\jmath}\right) & \equiv u_{\lambda}^{j} \eta^{\lambda} \quad\left(\bmod . H^{(l+k)}\right) .
\end{aligned}
$$

$F_{0}^{-1}$ is a homomorphism of $L^{\prime}$ into $L$ and the image of $L^{\left.\prime l^{\prime \prime}\right)}$ by $F_{0}^{-1}$ is in $L^{\left(l^{\prime}-k\right)}$ for any $l^{\prime}$. Therefore it follows by (11) and (12) that $\Omega_{\imath}=t_{i}^{j} \varphi_{j}$ and $\Pi_{\lambda}=u_{\lambda}^{j} \varphi_{j}$. 
$+u_{\lambda}^{\beta} \psi_{\sigma}$ form a subordination $s_{1}$ of $\mathbb{B}_{2}$ to $\mathbb{B}_{1}$. Since $F_{0}$ is the inverse of $\left(F^{-1}\right)_{0}$, we can construct, by the same method, a subordination $s_{2}$ of $\mathbb{B}_{3}$ to $\mathbb{B}_{2}$, which satisfies the required conditions. This finishes the proof.

By Prop. IV. 15 and 16, we have the following two theorems:

Theorem IV.9. Let $(\mathfrak{B}, \mathfrak{p})$ and $\left(\mathfrak{S}^{\prime}, \mathfrak{p}^{\prime}\right)$ be local transitive continuous pseudogroups. Denote by $G$ and $G^{\prime}$ parameter infinite Lie $(F)$-groups of $(\mathcal{B}, p)$ and $\left(\$^{\prime}, \mathfrak{p}^{\prime}\right)$, respectively. Then $G$ and $G^{\prime}$ are isomorphic if and only if $(\mathbb{B}, p)$ and $\left(\$^{\prime}, \mathfrak{p}^{\prime}\right)$ are equivalent.

Theorem VI.10. Let $G$ and $G^{\prime}$ be intinite Lie $(F)$-groups. Assume that there are local trasitive continuous pseudo-groups $(\mathbb{B}, \mathfrak{p})$ and $\left(\mathcal{B}^{\prime}, \mathfrak{p}^{\prime}\right)$ such that $G$ and $G^{\prime}$ are parameter groups of $(\mathfrak{S}, \mathfrak{p})$ and $\left(\mathbb{S}^{\prime}, \mathfrak{p}^{\prime}\right)$, respectively. Then $G$ and $G^{\prime}$ are convergently isomorphic if and only if $G$ and $G^{\prime}$ are isomorphic.

\section{ReFEREnCEs (Continued)}

[5] C. Ehresmann, Introduction à la théorie des structures infinitésimals et des pseudogroupes de Lie, Colloque International de Gémétrie Différentielle du C.N.R.S., 1953.

[6] M. Kuranishi, On E. Cartan's prolongations theorem of extericr differential systems, Amer. Journal of. Math. 1 (1957), pp. 1-47.

[7] M. Kuranishi, Lecture on exterior differential systems, Tata Institute of Fundamental Research, Bombay (In preparation).

\section{Mathematical Institute}

Nagoya University 